\title{
Chromatin remodeler HELLS maintains glioma stem cells through E2F3 and MYC
}

\author{
Guoxin Zhang, ${ }^{1}$ Zhen Dong, ${ }^{1}$ Briana C. Prager,, ${ }^{1,2}$ Leo J.K. Kim, ${ }^{1,2}$ Qiulian Wu, ${ }^{1}$ Ryan C. Gimple, ${ }^{1,2}$ \\ Xiuxing Wang, ${ }^{1}$ Shideng Bao, ${ }^{3}$ Petra Hamerlik, ${ }^{4,5}$ and Jeremy N. Rich ${ }^{1}$ \\ 'Division of Regenerative Medicine, Department of Medicine, University of California, San Diego, La Jolla, California, USA. \\ ${ }^{2}$ Department of Pathology, Case Western Reserve University School of Medicine, Cleveland, Ohio, USA. ${ }^{3}$ Department of \\ Stem Cell Biology and Regenerative Medicine, Lerner Research Institute, Cleveland Clinic, Cleveland, Ohio, USA. ${ }^{4}$ Danish \\ Cancer Society Research Center, Copenhagen, Denmark. ${ }^{5}$ Department of Drug Design and Pharmacology, Copenhagen \\ University, Copenhagen, Denmark.
}

\begin{abstract}
Clioblastomas, which contain stem cell-like glioblastoma stem cells (CSCs), are universally lethal cancers. While neural stem cells (NSCs) are usually quiescent, single-cell studies suggest that proliferating glioblastoma cells reside in the CSC population. Interrogating in silico glioma databases for epigenetic regulators that correlate with cell cycle regulation, we identified the chromatin remodeler HELLS as a potential target in glioblastoma. CSCs preferentially expressed HELLS compared with their differentiated tumor progeny and nonmalignant brain cells. Targeting HELLS disrupted CSC proliferation, survival, and self-renewal with induction of replication stress and DNA damage. Investigating potential molecular mechanisms downstream of HELLS revealed that HELLS interacted with the core oncogenic transcription factors, E2F3 and MYC, to regulate gene expression critical to CSC proliferation and maintenance. Supporting the interaction, HELLS expression strongly correlated with targets of E2F3 and MYC transcriptional activity in glioblastoma patients. The potential clinical significance of HELLS was reinforced by improved survival of tumor-bearing mice upon targeting HELLS and poor prognosis of glioma patients with elevated HELLS expression. Collectively, targeting HELLS may permit the functional disruption of the relatively undruggable MYC and E2F3 transcription factors and serve as a novel therapeutic paradigm for glioblastoma.
\end{abstract}

Authorship note: GZ and ZD contributed equally to this work.

Conflict of interest: The authors have declared that no conflict of interest exists.

Copyright: (c) 2019 American Society for Clinical Investigation

Submitted: November 12, 2018 Accepted: February 14, 2019 Published: April 4, 2019.

Reference information: $/ \mathrm{Cl}$ Insight 2019;4(7):e126140. https://doi. org/10.1172/jci.insight.126140.

\section{Introduction}

Glioblastoma (World Health Organization grade IV glioma) is the most prevalent and malignant primary, intrinsic brain tumor $(1,2)$. Current glioblastoma management entails maximal surgical resection followed by concurrent radiotherapy and chemotherapy with the oral methylator, temozolomide, followed by adjuvant temozolomide (3). Tumor treating fields and bevacizumab have been approved for glioblastoma treatment but provide marginal benefit in patient survival (4). While median survival of glioblastoma is commonly stated as 12-15 months, these results are from patients under the age of 70 who have a favorable performance status (3). Glioblastoma was among the first cancers studied by The Cancer Genome Atlas (TCGA) and ranks among the most thoroughly characterized of human cancers, yet this molecular understanding has translated poorly into meaningful survival benefit for glioblastoma patients.

Glioblastoma was previously designated as glioblastoma multiforme, due to its highly undifferentiated and heterogeneous histology. Phenotypic characterization of patient-derived xenografts and genetically engineered mouse models of glioblastoma has revealed that morphologic heterogeneity extends to a diversity of cellular phenotypes, with essential contributions from the tumor stroma and a diversity of tumor cells. Glioblastomas display heterogeneity of genetic aberrations and single-cell transcription (5-7) but also manifest cellular hierarchies that partially recapitulate developmental programs with stemlike tumor cells, called glioblastoma stem cells (GSCs) or brain tumor-initiating cells, at the apex (8). Although the precise cellular origin and identification of GSCs remains unresolved, glioblastomas have been one of the most reliable solid cancers for which cancer stem cells have been derived. GSCs are functionally defined through their ability to self-renew, sustain proliferation, and propagate tumors upon transplantation $(9,10)$. GSCs reside in perivascular and hypoxia niches and contribute to tumor growth 
through proliferation, promotion of angiogenesis, and invasion into normal brain (11-13). The clinical significance of GSCs is additionally supported by their resistance to DNA damage, which contributes to the futility of the conventional therapy and to the recurrence of the tumor following therapy (14). These features suggest GSCs as potential targets for novel therapy.

To date, a number of molecular targets that may be useful against GSCs have been derived, and early clinical trial development has been undertaken. Based on this background, we hypothesized that the sustained proliferative potential of GSCs may reveal novel molecular targets amenable to targeting, beyond the traditional cytotoxic modalities of ionizing radiation and chemotherapy, to which GSCs display relative resistance. Elevated mitotic indices are pathologic hallmarks of the transition from low-grade to high-grade gliomas, but the prognostic significance of proliferation has shown mixed results. Viewed through the prism of stem cell biology, proliferation in GSCs may be more informative than bulk tumor studies, as coexpression of GSC markers and proliferation in clusters are associated with poor prognosis (15). Further, single-cell analyses of gliomas suggest that proliferating cells within gliomas express stem cell regulatory programs, suggesting a correlation between cell proliferation and tumor stem cell maintenance (16). While traditionally cancer has been viewed as a disease caused by genetic mutations, accumulating evidence shows that epigenetic regulation also contributes to tumor initiation and progression $(17,18)$. Here, we interrogated key epigenetic regulators to further understand key processes that drive glioblastoma pathogenesis.

\section{Results}

HELLS is associated with glioma cell proliferation and is preferentially expressed by GSCs. Based on the strong association in single-cell RNA-seq between stem cell transcriptional profiles and cell cycle regulation in gliomas, we hypothesized that aberrant proliferative control may serve as a discovery tool for novel GSC targets. To identify active cycling cells, we interrogated the expression of a previously reported 55-gene panel of cells in the $\mathrm{G}_{2} / \mathrm{M}$ cell cycle phase (16) in glioma TCGA data sets, which confirmed an association between cell cycle control and histology, with glioblastoma expressing the highest levels of key cell cycle regulatory genes (Supplemental Figure 1A; supplemental material available online with this article; https://doi.org/10.1172/ jci.insight.126140DS1). Given the critical role of epigenetic regulation in glioblastoma biology, generally, and GSCs, specifically, we developed an 184-gene panel of genes that were determined to be writers, readers, editors, and erasers of epigenetic marks from a previously reported list of 212 epigenetic enzymes (19). We ranked the panel of epigenetic enzymes against the $\mathrm{G}_{2} / \mathrm{M}$ cell cycle signature, revealing the tightest positive correlation with targets that included genes previously associated with tumor grade (PCNA) or GSC regulation (EZH2, DNMT1, DNMT3B, and HDAC2) (20-22), findings that support the discovery strategy as being potentially useful to identify GSC targets (Figure 1A). One of the most tightly correlated epigenetic regulators with cell cycle regulators in glioblastoma patients was HELLS (helicase, lymphoid-specific; SWI/SNF2-related, matrix-associated, actin-dependent regulator of chromatin, subfamily A, member 6; also known as lymphoid-specific helicase [LSH]) (23). To inform greater granularity in the relationship between individual chromatin regulators and cell cycle genes, we mapped the epigenetic regulators with the highest positive and negative correlations with the full $\mathrm{G}_{2} / \mathrm{M}$ transcriptional signature against individual cell cycle regulatory genes, again revealing $H E L L S$ as one of the most tightly correlated genes with cell cycle control (Figure 1B). HELLS expression in glioma patients in TCGA data set strongly correlated with cell cycle gene expression ( $\mathrm{r}$ $\geq 0.7$; Supplemental Figure 1B). Given the recent molecular classification of gliomas, we mapped the expression of the top epigenetic regulatory genes correlated with proliferation across glioma samples in TCGA, with consideration of tumor transcriptional subtyping, IDH1/p53/PTEN mutational status, grade, age, and performance status, confirming that HELLS and the other genes positively correlated with cell cycle progression were highly enriched in older patients with worse performance status with IDH1 WT glioblastomas (Figure 1C). In single-cell RNA-seq data from 4 glioblastoma tumors, HELLS expression was enriched in SOX2+ glioblastoma cells (Figure 1, D and E).

Based on the enriched HELLS expression in SOX2 $2^{+}$glioblastoma cells and the correlation between $H E L L S$ expression and cell cycle regulators with the enrichment of cell cycle progression in glioma cells expressing stem cell programs, we interrogated the specificity of HELLS transcriptional control in GSCs. $H E L L S$ mRNA levels in GSCs were consistently higher in patient-derived GSCs relative to matched differentiated glioblastoma cells (DGCs) as well as a panel of nonmalignant brain cultures derived from epilepsy surgical resections (Figure 2A). To validate that preferential HELLS expression in GSCs is due to transcriptional control, we interrogated histone $\mathrm{H} 3$ lysine 27 acetyl (H3K27ac) deposition, which marks active 
promoters and enhancers $(24,25)$, in our patient-derived GSCs and matched DGCs, confirming elevated H3K27ac signal in the HELLS gene promoter of GSCs compared with that in DGCs (Supplemental Figure 1C). To confirm that the differential transcriptional regulation of HELLS in GSCs translated into elevated protein expression, we measured protein levels by immunofluorescent staining and immunoblotting and found increased HELLS levels in GSCs with minimal expression in DGCs and nonmalignant cultures (Figure 2, B-D). CDCA7, which is important for chromatin remodeling activity of HELLS (26), was also enriched in GSCs compared with DGCs (Supplemental Figure 1, E and F). Thus, HELLS is strongly associated with glioblastoma cell proliferation and highly expressed in GSCs.

HELLS maintains GSC proliferation and self-renewal. To examine the functional role of HELLS in GSCs, we targeted HELLS expression using 2 nonoverlapping shRNAs against HELLS (shRNA.1744 and shRNA.1308, designated by the start site of the shRNA on the transcript) compared with a control shRNA sequence that does not target any mammalian gene (shCONT). HELLS was dispensable for nonneoplastic brain cells, as measured by a luminescence-based cell viability assay (Supplemental Figure 2, A-D). Targeting HELLS moderately reduced the proliferation of DGCs (Supplemental Figure 2, E-H). In striking contrast, targeting HELLS potently reduced proliferation in multiple patient-derived GSCs (Figure 3, A-F). Cancer stem cells are functionally defined, in part, by the ability to self-renewal, for which sphere formation serves as a surrogate, albeit with caveats. Targeting HELLS expression potently reduced the self-renewal of 3 patient-derived GSCs in limiting dilution (Figure 3, G-L). Collectively, GSCs display dependence of HELLS to maintain proliferation and self-renewal.

Targeting HELLS induces replication stress and DNA damage. Although HELLS was named based on presumed helicase function, its function is likely distinct, as HELLS is a member of the switch/sucrose nonfermenting (SWI2/SNF2) superfamily, which provide the energy necessary for chromatin remodeling through the hydrolysis of ATP (27). The SWI2/SNF2 superfamily is divided into 4 distinct subfamilies based on the presence of additional functional domains: SWI/SNF, imitation switch (ISWI), chromodomain helicase DNA-binding (CHD), and inositol 80 (INO80) (27). To determine if the pattern of HELLS expression is generally held by other family members, we interrogated expression patterns of a panel of additional SWI2/SFN2 superfamily members (CHD2, CHD3, CHD4, and CHD5) in relation to cell cycle regulatory genes in glioblastomas. Whereas $H E L L S$ is tightly correlated to the expression of core regulators of cell cycle progression, the other superfamily members had no correlation (Supplemental Figure 3A).

To determine if HELLS is a driver of the proliferation program, we performed ChIP-qPCR on GSCs and found that HELLS bound to the promoter regions of cell cycling genes, including MKi67, PCNA, CCNB1, and CDC6 (Supplemental Figure 3, B and C). Furthermore, HELLS knockdown reduced expression of these genes (Supplemental Figure 3, D and E). Combined with the loss of GSC proliferation observed after HELLS knockdown, these observations suggest that HELLS supports GSC cell cycle progression.

As chromatin remodeling plays an active role in DNA repair and genome stability maintenance (27), we assessed whether the disruption of HELLS expression induces replication stress and DNA damage. Phosphorylated replication protein A (pRPA, Ser32) marks replication stress (28). Targeting HELLS expression increased pRPA foci, as quantified by immunofluorescence (Figure 4, A and B). The percentage of $\mathrm{pRPA}^{+}$cells, with a threshold of at least 5 foci/cell, increased upon HELLS knockdown in GSCs (Figure 4C). Consistent with the immunofluorescent staining, immunoblotting also showed increased pRPA levels in GSCs after HELLS knockdown (Figure 4D). Severe replication stress may cause DNA double-strand breaks (DSBs) (29). Targeting HELLS expression in GSCs induced DSBs, as measured by immunofluorescent staining for $\gamma \mathrm{H} 2 \mathrm{AX}$ (Figure 4, E and F). Quantification of DSBs, as measured by the fraction of cells with at least $5 \gamma \mathrm{H} 2 \mathrm{AX}$ foci, confirmed that DSBs increased in GSCs upon HELLS knockdown (Figure $4 \mathrm{G}$ ), which was further confirmed by flow cytometry assays and immunoblotting for $\gamma \mathrm{H} 2 \mathrm{AX}$ (Figure $4 \mathrm{H}$ and Supplemental Figure 3F). Thus, loss of HELLS expression in GSCs induces genotoxic stress.

Targeting HELLS reduces cell cycle progression and induces apoptosis in GSCs. Induction of DNA damage may lead to the activation of cell cycle checkpoints and arrest of cell cycle. Therefore, we examined HELLS regulation of GSC cell cycle progression using a 5-ethynyl-2-deoxyuridine (EdU) incorporation assay. Targeting HELLS reduced the percentage of $\mathrm{EdU}^{+}$(actively proliferating) cells in 2 patient-derived GSCs, as measured by flow cytometry (Figure 5, A-D). To confirm the observations from EdU-based flow cytometry, the Ki-67 labeling index was measured by immunofluorescence in GSCs following HELLS knockdown, confirming substantial reduction in proliferation with loss of HELLS expression (Figure 5, E-G). Collectively, these results demonstrate that HELLS is required for GSC proliferation. 
A

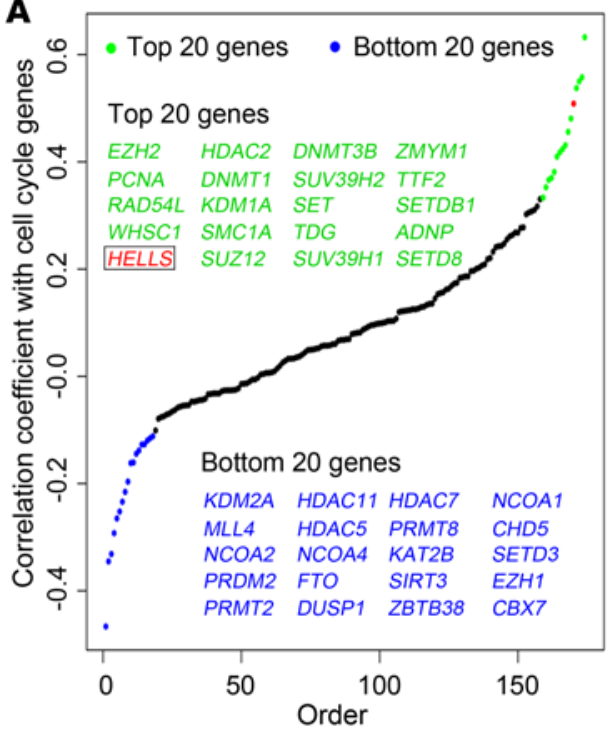

B

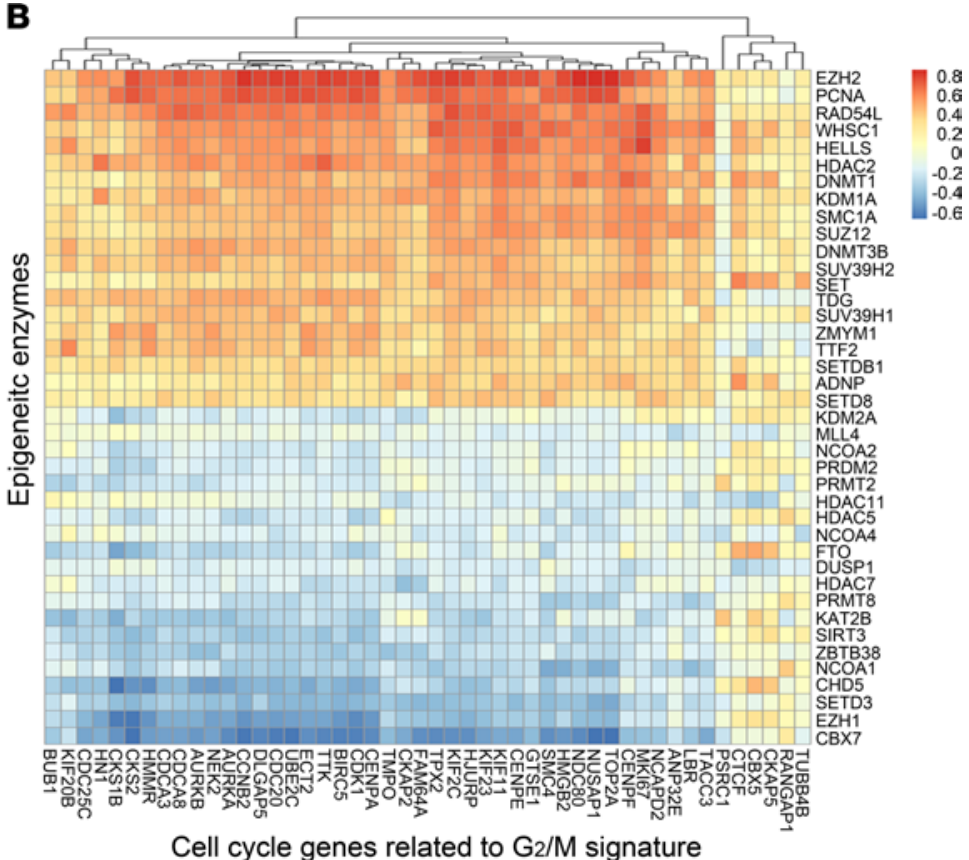

C

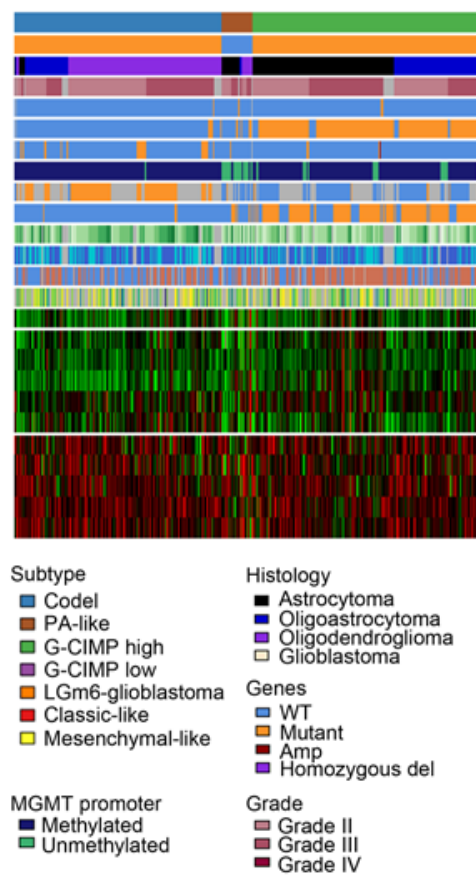

\section{D}

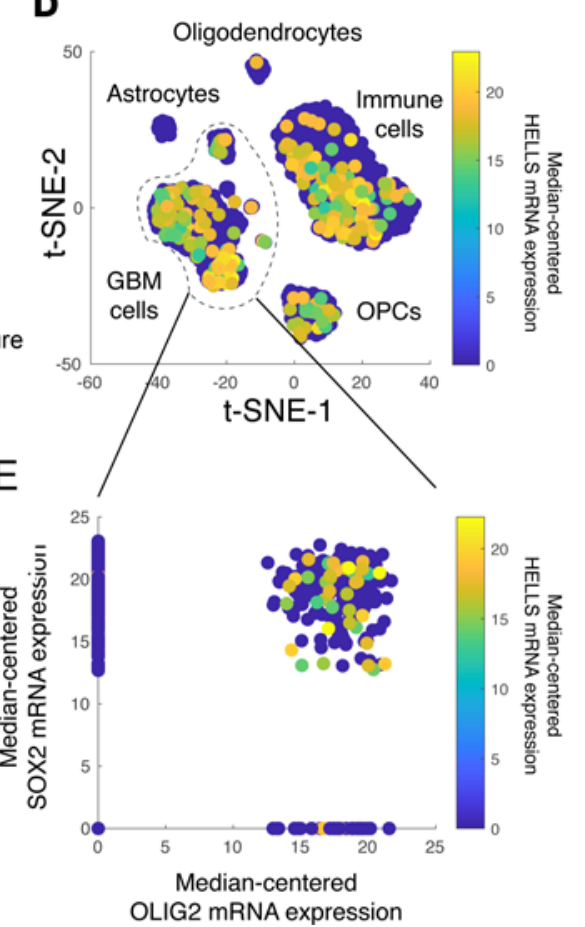

Figure 1. Proliferating glioma cells express HELLS. (A) Correlation between mRNA expression levels of chromatin regulators and $\mathrm{G}_{2} / \mathrm{M}$ cell cycle signature in glioblastoma patients. The top 20 (green) and bottom 20 (blue) epigenetic regulators are listed. HELLS is labeled in red. (B) Heatmap displaying correlations between epigenetic regulators and individual $\mathrm{G}_{2} / \mathrm{M}$ signature gene expression in glioblastoma patients. The top 20 and bottom 20 chromatin regulators are displayed. (C) RNA-seq, whole exome, and clinical phenotype data were aggregated from TCGA glioblastoma (GBM) and low-grade glioma (LGG) data set to visualize the expression patterns of top 5 and bottom 5 epigenetic regulators identified in A. "Codel," codeletion of chromosomes $1 p$ and 19q; PA-like, pilocytic astrocytoma-like; CIMP, glioma-CpG island methylator phenotype; LGm6-CBM, a subgroup of glioma enriched for histologic lowgrade gliomas that also contains a subset of tumors with GBM-defining histologic criteria; KPS, Karnofsky performance status. (D and E) HELLS expression is enriched in SOX2+ glioblastoma cells in bulk tumor single-cell RNA-seq data sets. (D) t-Distributed stochastic neighbor embedding (t-SNE) plot of combined single-cell RNA-seq data from 4 glioblastoma tumors. Each dot represents a single cell with HELLS mRNA expression denoted by the color map. (E) Scatter plot of SOX2 and OLIC2 mRNA expression among glioblastoma tumor cells. Each dot represents a single cell with HELLS mRNA expression denoted by the color map. 
A

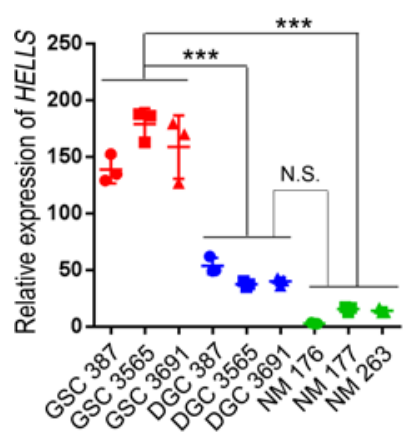

B
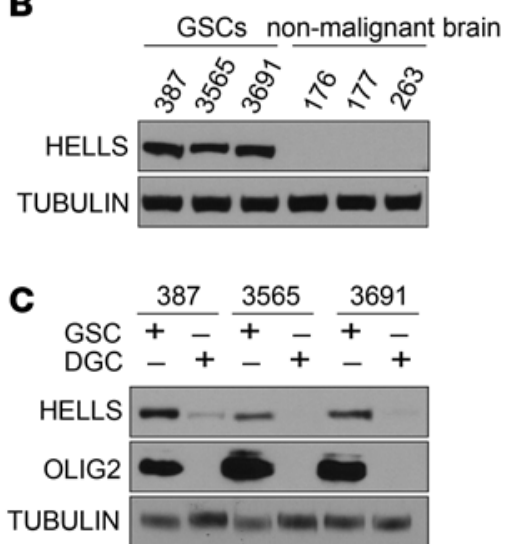

D

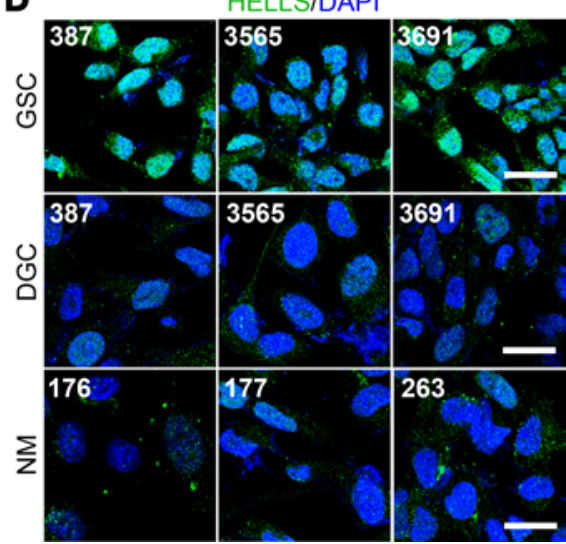

Figure 2. GSCs preferentially express HELLS. (A) qPCR analysis of HELLS mRNA levels in matched CSCs and DGCs (387, 3565, and 3691) and nonmalignant brain cultures $(176,177$, and 263$)$. At least 3 independent experiments were performed. Data are presented as mean $\pm S D$. ${ }^{* *} P<0.01$, ${ }^{* * *} P<0.001$, by 1 -way ANOVA with Tukey's multiple comparisons test. (B) Western blot for HELLS protein in GSCs (387, 3565, and 3691) and nonmalignant brain cultures (176, 177, and 263). Tubulin was used as a loading control. Three independent experiments were performed. (C) Western blot for HELLS protein in matched GSCs and DGCs (387, 3565, and 3691) and nonmalignant brain cultures (176, 177, and 263). OLIG2 was used as a CSC marker. Tubulin was used as a loading control. Three independent experiments were performed. (D) Immunofluorescent staining of HELLS in matched GSCs and DCCs (387, 3565, and 3691) and nonmalignant brain cultures $(176,177$, and 263$)$. HELLS signals are shown as green, and DAPI as blue. Three independent experiments were performed. Scale bars: $20 \mu \mathrm{m}$.

Given that severe DNA damage may cause cell death, we interrogated the effect of HELLS on GSC survival. Flow cytometric quantification of annexin V/propidium iodide staining revealed that HELLS knockdown potently induced cell death (Figure 6, A-D). To address the mode of cell death, cleaved caspase-3, a marker for apoptosis, was detected with immunofluorescent staining. Consistent with results from annexin V staining-based flow cytometry assays, GSCs transduced with shCONT showed limited cleaved caspase-3, while GSCs with HELLS knockdown showed prominent cleaved caspase-3 staining (Figure 6, E-G), which was confirmed by immunoblotting for cleaved caspase-3 and the cleavage of polyADP ribose polymerase (PARP) (Figure 6, H and I). Taken together, these data indicate that HELLS knockdown induces GSC apoptosis in association with severe replication stress and DNA DSBs.

HELLS correlates with E2F3 and MYC targets in glioblastoma patients. To interrogate potential molecular mechanisms underlying HELLS control of cell proliferation, whole genome expression data from glioblastoma patients were analyzed. We stratified glioblastoma patients into 2 groups based on HELLS expression (top quartile vs. bottom quartile) (Supplemental Figure 4A). Gene ontology analysis showed that the differentially expressed genes between the HELLS ${ }^{\text {hi }}$ and HELLS ${ }^{\text {lo }}$ groups were strongly associated with cell cycle progression (Supplemental Figure 4, B-E). Given that HELLS functions without known direct transcriptional activity, HELLS could interact with transcriptional factors to regulate the expression of genes that are important for cell cycle progression. GSEA for the differentially expressed genes between HELLS ${ }^{\text {hi }}$ and HELLS ${ }^{\text {lo }}$ groups showed enriched gene sets, including E2F targets and MYC targets (Supplemental Figure 4, F-I). E2F3 controls cell proliferation and embryonic development $(30,31)$ and interacts with HELLS in colon cancer cells (32). E2F3 targets and MYC targets were preferentially expressed in HELLS ${ }^{\text {hi }}$ patient tumors (Supplemental Figure 5A). E2F3 and MYC targets were strongly correlated with HELLS expression (Supplemental Figure 5B). Collectively, these glioblastoma patient data from TCGA showed that E2F3 targets and MYC targets were enriched in differentially expressed genes between HELLS ${ }^{\text {hi }}$ and HELLS ${ }^{\text {lo }}$ tumors of glioblastoma patients.

RNA-seq data support interactions between HELLS and E2F3 and MYC. To understand the molecular mechanisms of HELLS functions in GSCs, RNA-seq was performed on 2 different GSCs transduced with either shCONT or shHELLS. While 2 GSCs clustered into different groups, cells transduced with shHELLS clustered together (Figure 7A). Unsupervised clustering of each RNA-seq sample with principle component analysis showed that HELLS knockdown altered GSC transcriptional profiles consistently (Figure 7B). Targeting HELLS expression induced similar transcriptional changes in each model, with modest differences between these 2 models (Figure 7C). GSEA identified molecular pathways associated with HELLS, revealing enrichment of gene sets related to E2F targets, MYC targets, and the $\mathrm{G}_{2} / \mathrm{M}$ checkpoint (Figure 7, $\mathrm{D}-\mathrm{H})$. Given the strong correlation between HELLS and E2F3 in glioblastoma patients, these data support the interactions between HELLS and E2Fs as well as HELLS and MYC. 
A

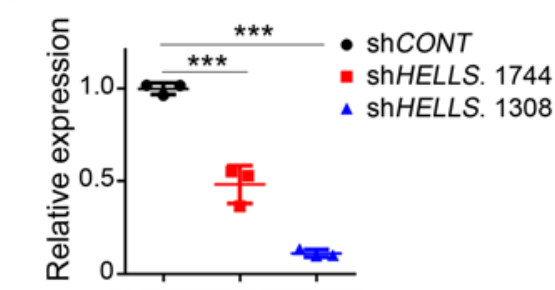

HELLS

TUBULIN
B

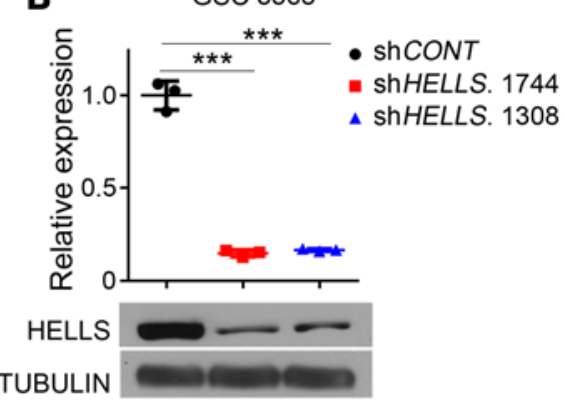

E

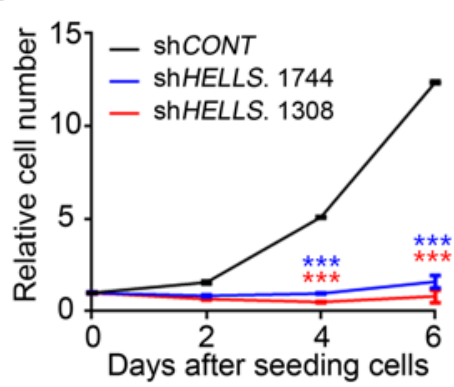

H

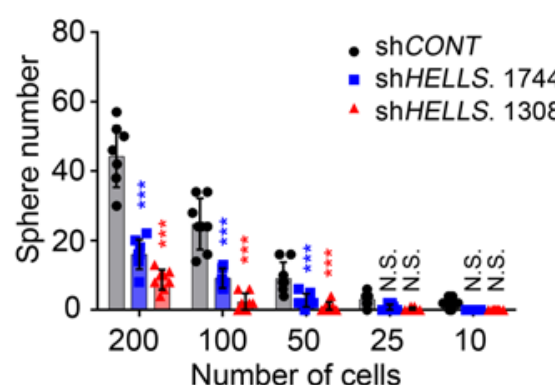

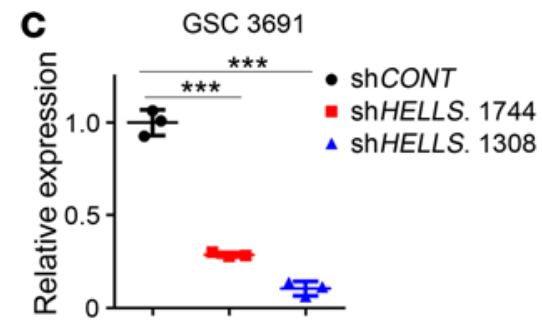

TUBULIN

$\mathbf{F}$

GSC 3691

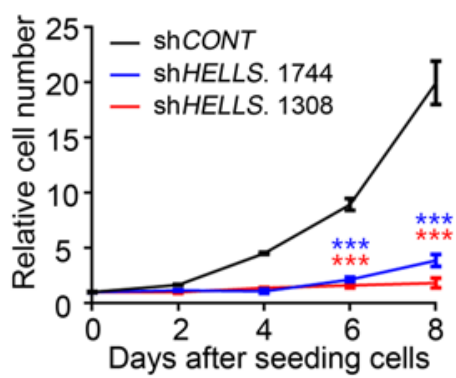

I

GSC 3691

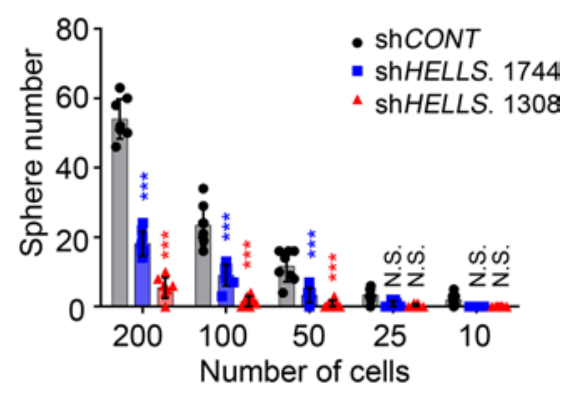

L

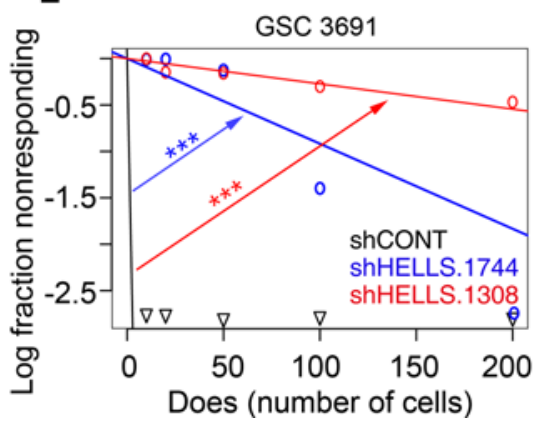

Figure 3. HELLS maintains GSC proliferation and self-renewal. (A-C) qPCR analysis of HELLS mRNA and immunoblot for HELLS protein levels after shRNA-mediated HELLS knockdown in GSC 387 (A), 3565 (B), and 3691 (C). Three independent experiments were performed. Data are presented as mean \pm SD. ${ }^{* * *} P<0.001$, by 1-way ANOVA with Tukey's multiple comparison test. (D-F) CellTiter-Glo assay after HELLS knockdown in GSC 387 (D), 3565 (E), and 3691 (F). Three independent experiments were performed. Data are presented as mean \pm SD. ${ }^{* *} P<0.001$, by 2-way ANOVA with Tukey's multiple comparisons test. (G-I) Sphere numbers of GSC $387(\mathbf{G}), 3565$ (H), and 3691 (I) after HELLS knockdown. Three independent experiments were performed. Data are presented as mean $\pm \mathrm{SD} .{ }^{*} P<0.05,{ }^{* *} P<0.001$, by 2 -way ANOVA with Tukey's multiple comparisons test. (J-L) Limited dilution assay after HELLS knockdown in GSC $387(\mathrm{~J}), 3565$ (K), and 3691 (L). Three independent experiments were performed. Data are presented as mean \pm SD. ${ }^{* * *} P<0.001$, by $\chi^{2}$ test for pair-wise differences. 

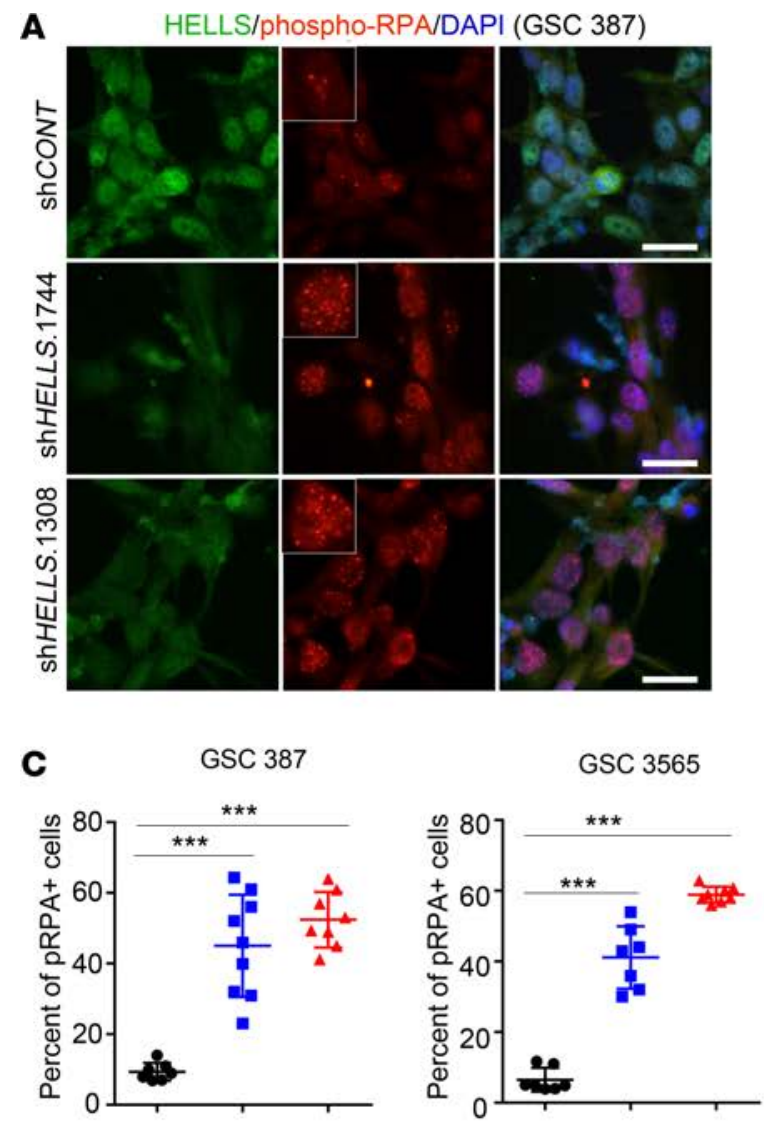

E
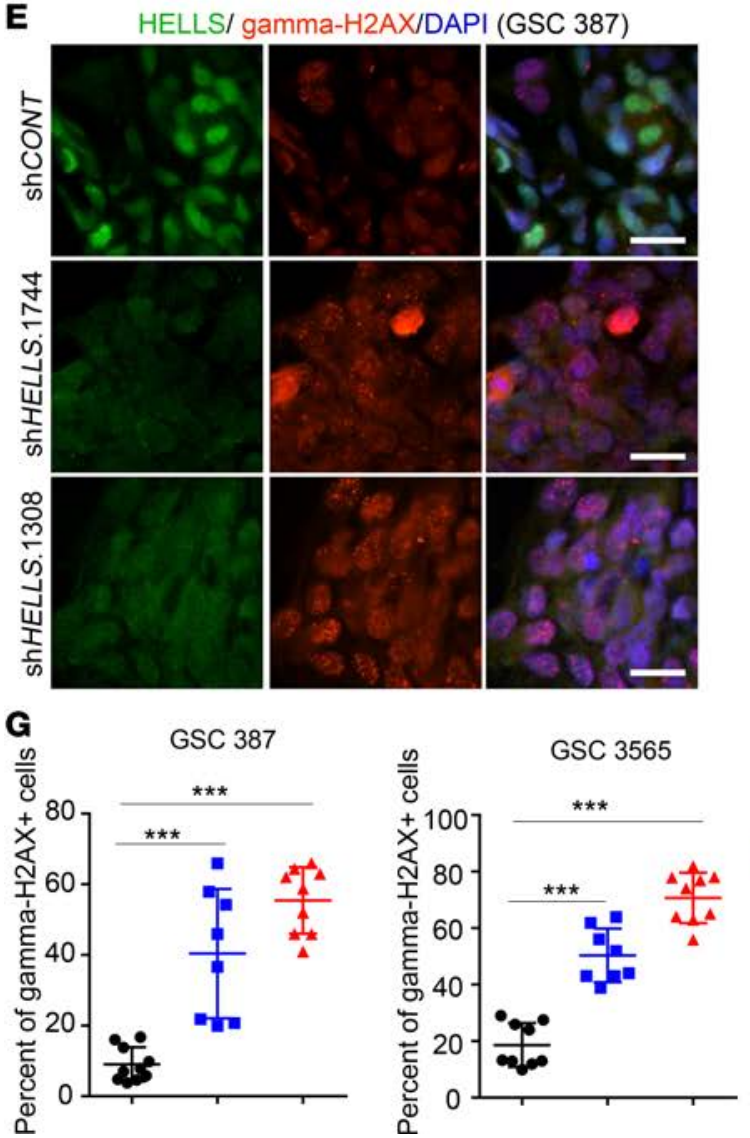

B HELLS/phospho-RPA/DAPI (GSC 3565)
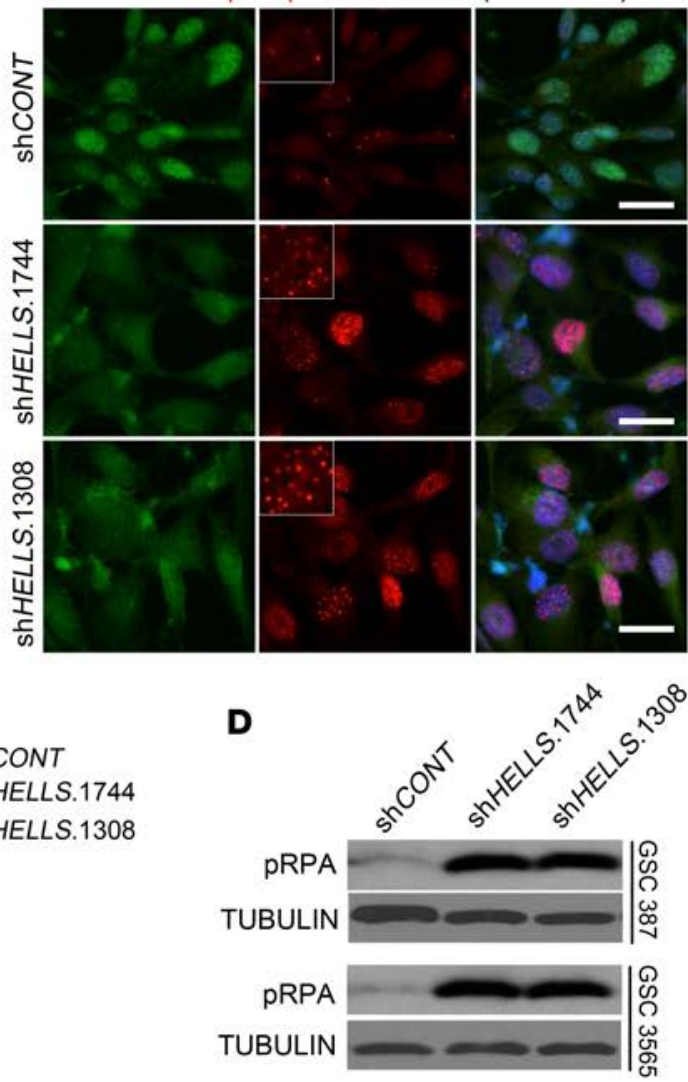

F HELLS/ gamma-H2AX/DAPI (GSC 3565)

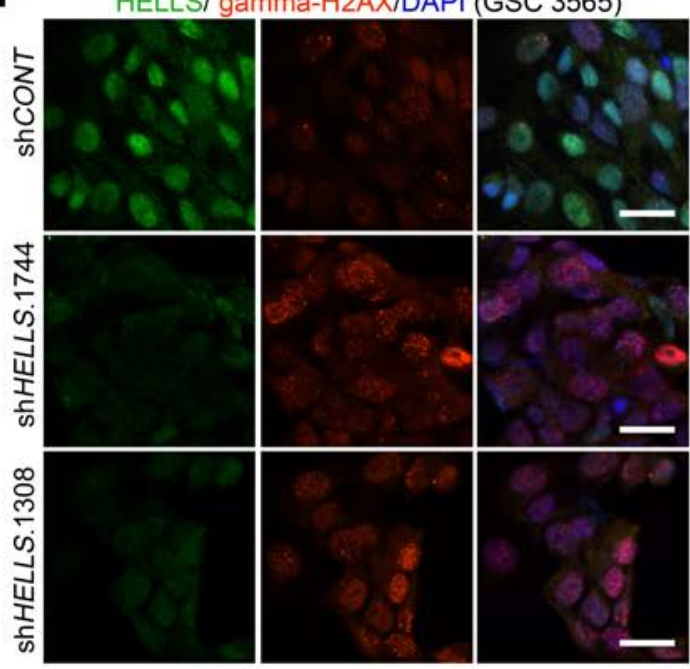

H

- $\operatorname{shCONT}$

- shHELLS.1744

A shHELLS.1308

Gamma-H2AX TUBULIN Gamma-H2AX TUBULIN 
Figure 4. Targeting HELLS induces replication stress and DNA damage. (A and B) Immunofluorescent staining of HELLS and phosphorylated RPA32/ RPA2 (pRPA) in GSC 387 (A) and 3565 (B) after knocking down HELLS expression. HELLS signals are shown as green and pRPA as red. DAPI is shown as blue. Three independent experiments were performed. Scale bars: $20 \mu \mathrm{m}$. (C) Quantification of cells with $\geq 5$ foci of phosphorylated RPA32/RPA2 foci in GSC model 387 and 3565 after HELLS knockdown. Data are presented as mean \pm SD. ${ }^{* *} P<0.001$, by 1-way ANOVA with Tukey's multiple comparisons test. shCONT, $n$ = 7; shHELLS.1744, $n$ = 9; shHELLS.1308, $n=8$. (D) Immunoblot for phosphorylated RPA32/RPA2 in GSC 387 and 3565 after HELLS knockdown. Tubulin was used as input. Three independent experiments were performed. (E and F) Immunofluorescent staining for HELLS and $\gamma \mathrm{H} 2 \mathrm{AX}$ in GSC 387 (E) and 3565 (F) after knocking down HELLS expression. HELLS signals are shown as green and $\gamma \mathrm{H} 2 \mathrm{AX}$ as red. DAPI was used for nucleus staining and is shown as blue. Three independent experiments were performed. Scale bars: $20 \mu \mathrm{m}$. (G) Quantification of cells with $\geq 5$ foci of H2AX foci in GSC model 387 and 3565 after HELLS knockdown. Data are presented as mean $\pm \mathrm{SD}$. ${ }^{* *} P<0.001$, by 1-way ANOVA with Tukey's multiple comparisons test. shCONT, $n$ = 7; shHELLS.1744, $n=7$; shHELLS.1308, $n=8$. (H)Immunoblot for $\gamma$ H2AX in CSC 387 and 3565 after HELLS knockdown. Tubulin was used as input. Three independent experiments were performed.

HELLS interacts with E2F3 and MYC to regulate gene expression. To directly interrogate HELLS interaction with E2F3 in GSCs, coimmunoprecipitation assays were performed. E2F3 has 2 different isoforms, E2F3a and E2F3b. Co-overexpression of FLAG-tagged HELLS and HA-tagged E2F3a or HA-tagged E2F3b in GSCs demonstrated that HELLS interacted with both E2F3a and E2F3b (Figure 8A). E2F3 and HELLS bound the same regions of E2F3 target gene promoters in 2 patient-derived GSCs using ChIP-qPCR assays (Figure 8B). Given their interactions, HELLS may contribute to E2F3 binding to its target genes in GSCs. Using ChIP-qP$\mathrm{CR}$ assays, enrichment of E2F3 in the promoter regions of its target genes decreased 36 hours after HELLS knockdown in 2 GSCs, while no decrease in E2F3 protein was observed, although E2F3 protein expression decreased slightly 12 hours later (Figure 8C and Supplemental Figure 6, A and B). The decreased binding of E2F3 after HELLS knockdown was further confirmed by the decreased enrichment of FLAG-tagged E2F3a or FLAG-tagged E2F3b at target gene promoters in GSCs expressing FLAG-tagged E2F3s (Supplemental Figure 6, C and D). Consistent with the decrease of E2F3 binding, all examined E2F3 target genes showed decreased expression after HELLS knockdown in both GSCs (Supplemental Figure 6E).

To determine whether decreased expression of E2F3 target genes is responsible for the phenotypes caused by HELLS knockdown in GSCs, E2F3 expression was knocked down in GSCs (Supplemental Figure 6F). GSC proliferation was impaired after E2F3 knockdown, suggesting that decreased expression of E2F3 target genes after HELLS knockdown may contribute to the subsequent phenotypes (Supplemental Figure 6G). However, E2F3 overexpression only partially compensated for HELLS knockdown in GSCs (Supplemental Figure $6 \mathrm{H}$ ), suggesting that more partners of HELLS are involved. In summary, HELLS interacts with E2F3 in GSCs, which contributes to E2F3 target gene expression by promoting binding of E2F3 to its targets.

MYC is another candidate that can interact with HELLS in GSCs. To test this hypothesis, GSCs expressing FLAG-tagged HELLS or FLAG-tagged GFP were established with 2 patient-derived GSCs. FLAG-HELLS, but not FLAG-GFP, bound MYC in both GSCs (Figure 8D). Endogenous HELLS also interacted with MYC in GSCs (Figure 8E). MYC and HELLS cobound to promoter regions of MYC target genes, as measured by ChIP-qPCR (Figure 8F). After HELLS knockdown, MYC expression was maintained in GSC 387 while it was slightly decreased in GSC 3565 (Supplemental Figure 7A). However, binding of MYC to its targets decreased in both GSCs (Figure 8G). As a consequence, targeting HELLS expression decreased expression of MYC target genes in GSCs, suggesting that the HELLS-MYC interaction contributes to MYC activity (Supplemental Figure 7B). However, MYC overexpression only partially rescued the HELLS knockdown in GSCs (Supplemental Figure 7, C and D).

HELLS is required for in vivo glioblastoma growth and informs prognosis. Our in vitro data indicated that HELLS is important for GSC maintenance. As in vivo tumor growth is the most important functional GSC assay, we interrogated the contribution of HELLS for in vivo tumor growth using orthotopic xenografting. Equal numbers of GSCs transduced with shRNAs targeting HELLS or nontargeting control (shCONT) were implanted intracranially into immunocompromised mice. Mice bearing ortotopic GSCs transduced with 1 of 2 shHELLS survived substantially longer than mice bearing GSCs transduced with shCONT (Figure 9A). Histologic staining of tumor-bearing brains collected 20 days after GSC transplantation revealed obvious tumor masses in mice bearing shCONT-transduced GSCs, while almost no tumor mass was found in mice injected with HELLS knockdown GSCs (Figure 9B). The prolonged survival indicated that HELLS was required for tumor growth in vivo.

To explore the functional importance of cell cycle progression and HELLS in glioma patients, expression levels were examined across different grades and subtypes of gliomas. The $\mathrm{G}_{2} / \mathrm{M}$ signature was elevated in glioblastoma compared with that in nonmalignant brain and tumors of lower grades 
A

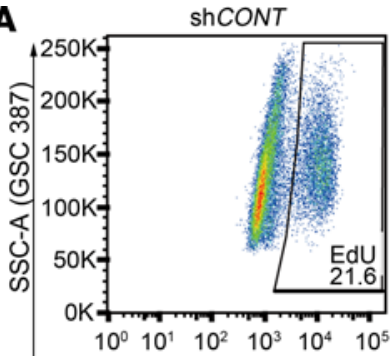

C

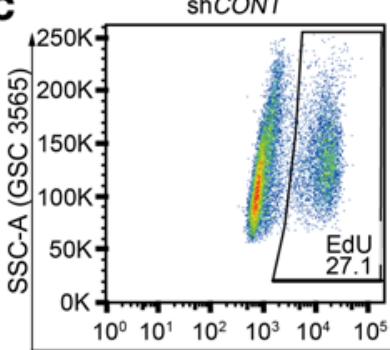

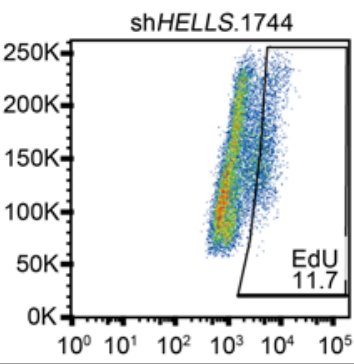

EdU (Blue 515-A)

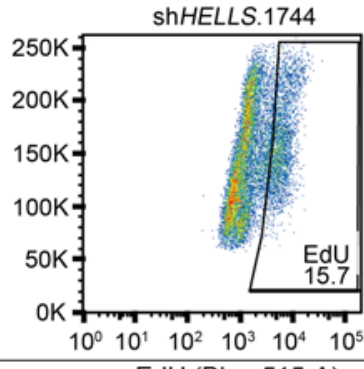

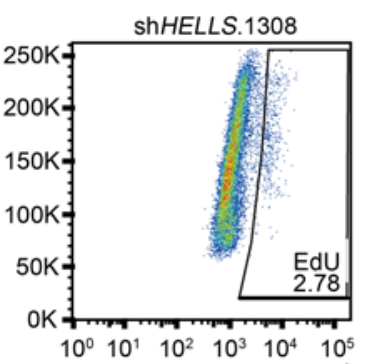

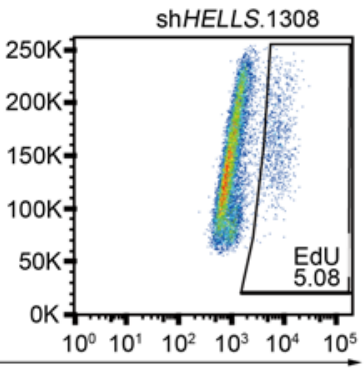

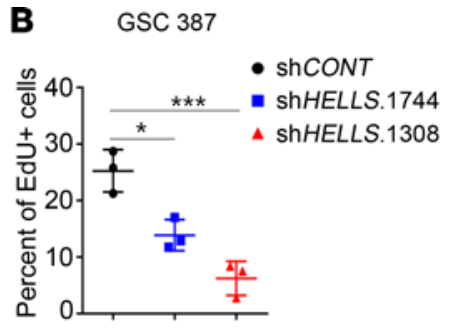

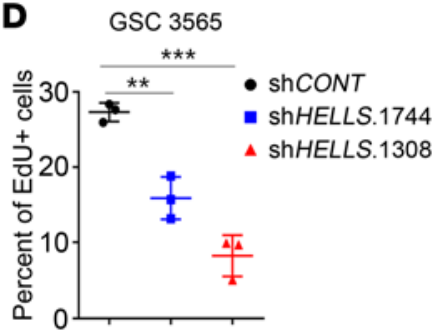

E

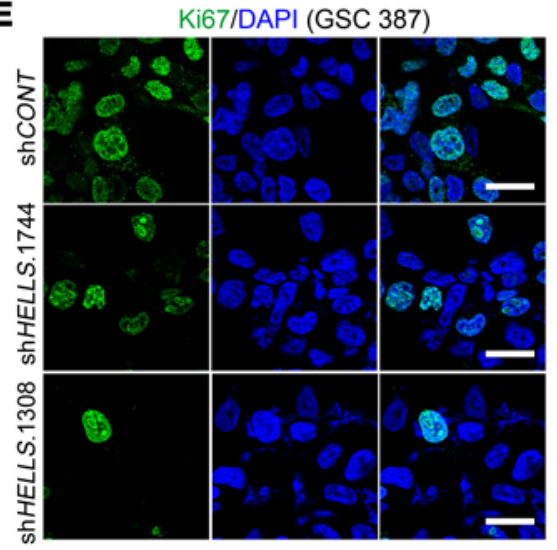

$\mathbf{F}$

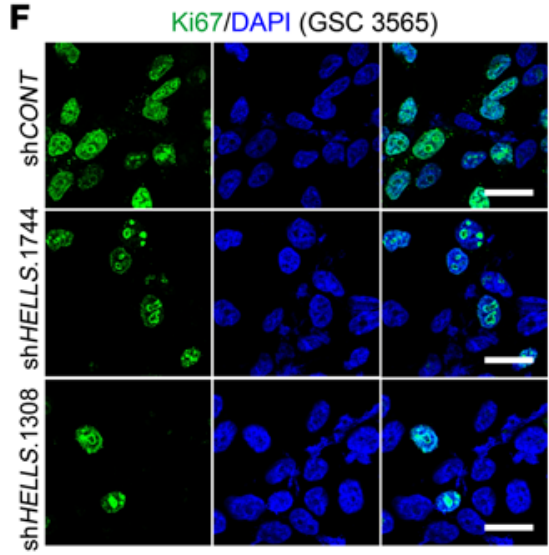

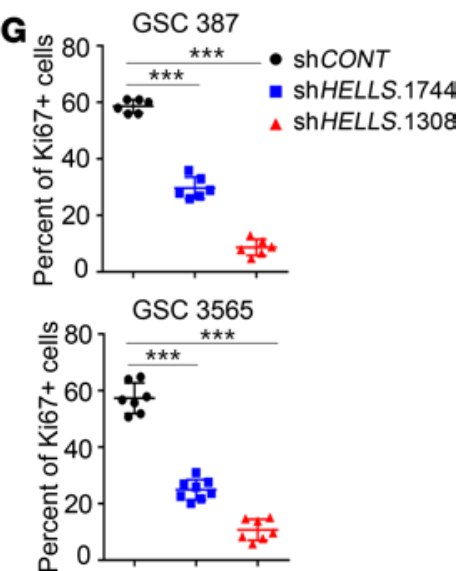

Figure 5. Targeting HELLS reduces GSC cell cycle progression. (A) Flow cytometry of EdU incorporation after 2-hour incubation with $10 \mu M$ EdU in GSC 387 analyzed 48 hours after HELLS knockdown. The $y$ axis was gated by SSC, and the $x$ axis was gated by EdU signals. Three independent experiments were performed. (B) Quantification of EdU+ cells from A. Data are presented as mean \pm SD. ${ }^{*} P<0.05,{ }^{* *} P<0.001$, by 1-way ANOVA with Tukey's multiple comparisons test. Three biologic replicates were used. (C) Flow cytometry of EdU incorporation assays after 2-hour incubation with $10 \mu \mathrm{M}$ EdU in GSC 3565 analyzed 48 hours after HELLS knockdown. The $y$ axis was gated by SSC, and the $x$ axis was gated by EdU signals. Three independent experiments were performed. (D) Quantification of EdU+ cells from C. Data are presented as mean $\pm \mathrm{SD}$. ${ }^{* *} P<0.01,{ }^{* *} P<0.001$, by 1-way ANOVA with Tukey's comparison test. Three biologic replicates were used. (E and F) Ki67 staining in GSC 387 (E) and 3565 (F) after HELLS knockdown. Ki67 signals are shown as green and DAPI as blue. Three independent experiments were performed. Scale bars: $20 \mu \mathrm{m}$. (C)Quantification of Ki67+ cells in GSC 387 (top) and 3565 (bottom) after HELLS knockdown. Data are presented as mean \pm SD. ${ }^{* *} P<0.001$, by 1-way ANOVA with Tukey's multiple comparisons test. For GSC 387: shCONT, $n=6$; $\operatorname{shHELLS.1744,~} n=6$; shHELLS.1308, $n=6$. For GSC 3565: shCONT, $n=7$; shHELLS.1744, $n=8 ;$ shHELLS.1308, $n=7$.

(Supplemental Figure $8, \mathrm{~A}$ and $\mathrm{B}$ ). Further, the $\mathrm{G}_{2} / \mathrm{M}$ signature informed poor prognosis across glioma types but was not prognostic within glioblastoma, as expected (Supplemental Figure 8, C and D). HELLS expression and E2F3 and MYC target genes were also strongly associated with IDH1 status in glioblastoma (Figure 9C). HELLS expression was elevated in glioblastoma tissues compared with that in nonmalignant brain tissues (Supplemental Figure 8E). HELLS expression correlated with histology, with glioblastoma having the highest expression (Figure 9D) and tumor grade (Figure 9E). HELLS expression portended a poor prognosis for glioma patients in TCGA and REMBRANDT with worse survival (Figure 9F and Supplemental Figure 8F). Patients with WT IDH glioblastoma were enriched in the HELLS ${ }^{\text {hi }}$ group compared with the HELLS ${ }^{\text {lo }}$ group (Figure 9F), suggesting a correlation between HELLS and WT IDH. E2F3 and MYC are overexpressed in other tumor types in which cancer stem cells have been described, with HELLS and E2F3/MYC correlated in several other tumors, although not uni- 
A

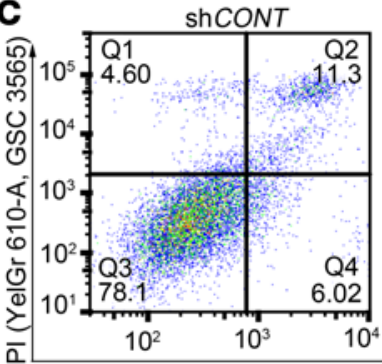

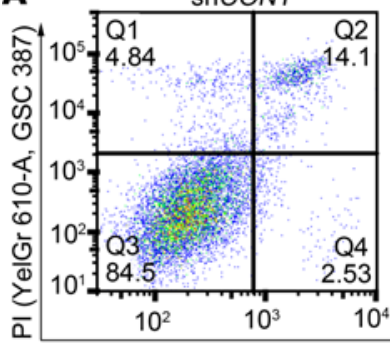
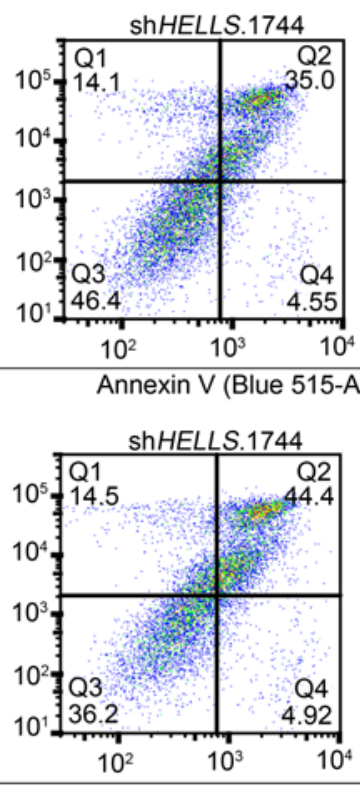

Annexin V (Blue 515-A)
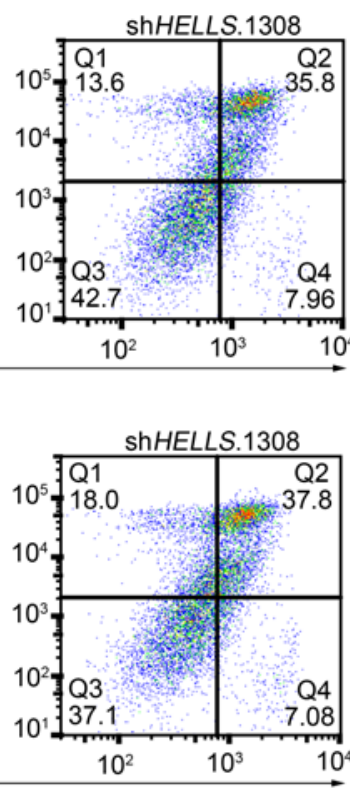

$\mathbf{F}$
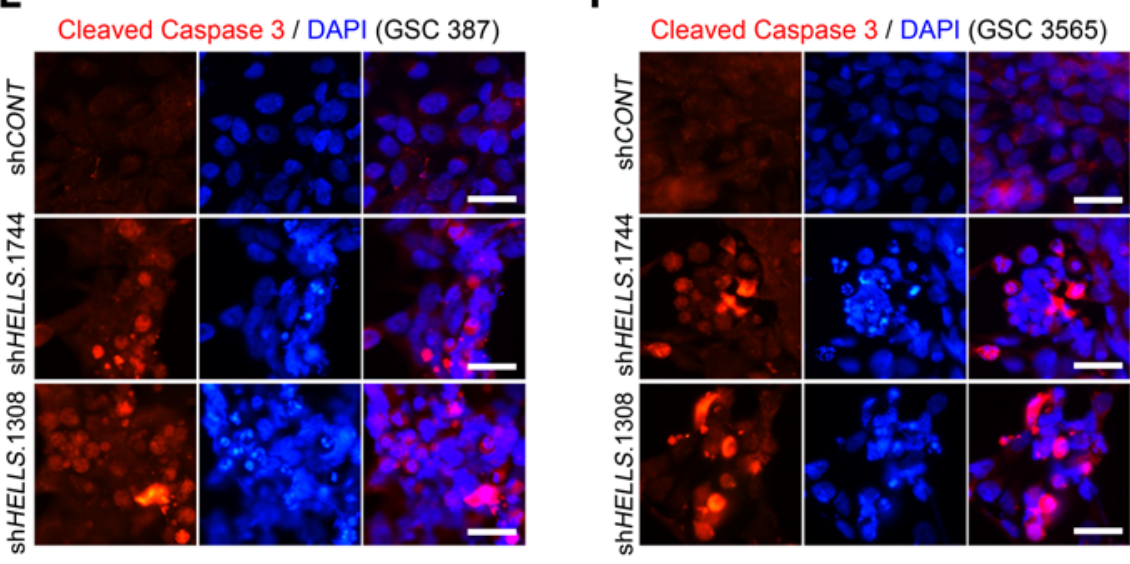

B

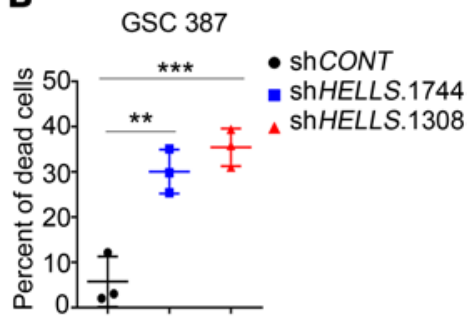

H

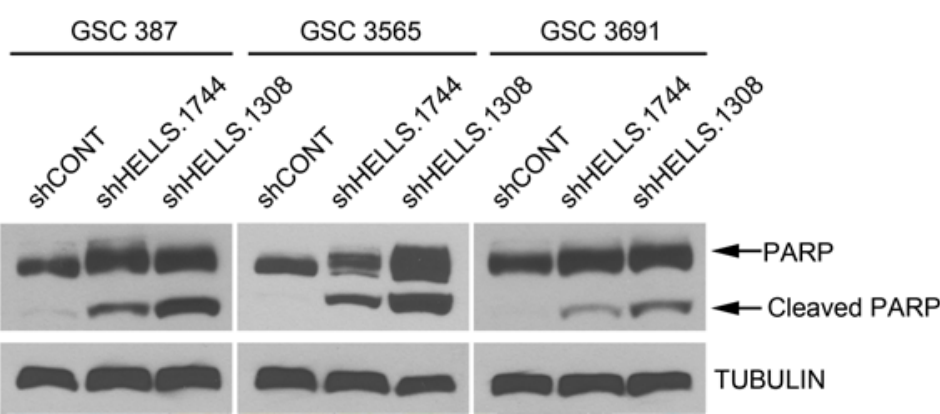

D

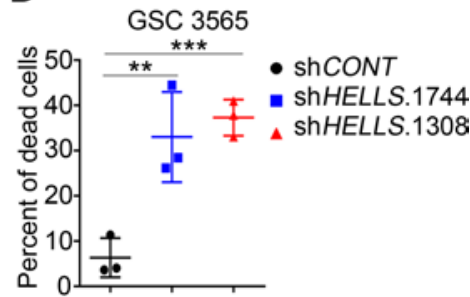

G

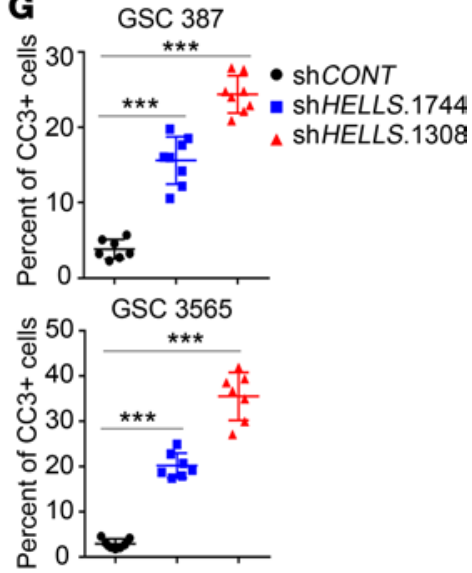

I

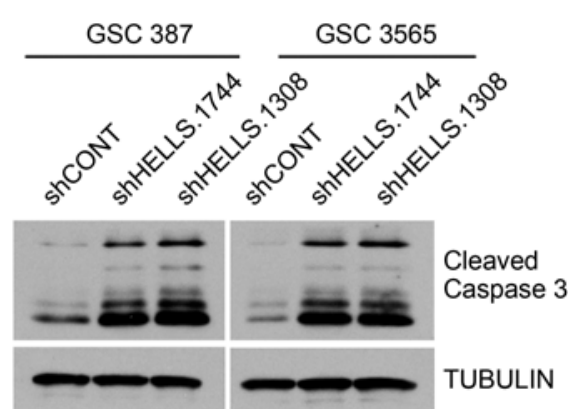

Figure 6. Disruption of HELLS expression induces GSC apoptosis. (A) Flow cytometry of annexin V/propidium iodide (annexin V/PI) staining in GSC 387 analyzed 48 hours after HELLS knockdown. Signals from PI are shown on the $y$ axis, and those from annexin $V$ are shown on the $x$ axis. Three independent experiments were performed. (B) Quantification of annexin V/PI double-positive populations from $\mathbf{A}$. Three independent experiments were performed. Data are presented as mean $\pm \mathrm{SD}$. ${ }^{* *} P<0.01,{ }^{* *} P<0.001$, by 1-way ANOVA with Tukey's multiple comparisons test. (C) Flow cytometry of annexin V/PI staining in GSC 3565 analyzed 48 hours after HELLS knockdown. Signals from PI are shown on the $y$ axis, and those from annexin $V$ are shown in $x$ axis. Three independent experiments were performed. (D) Quantification of annexin V/PI double-positive populations from C. Three independent experiments were performed. Data are presented as mean $\pm \mathrm{SD}$. ${ }^{* *} P<0.01$, ${ }^{* *} \mathrm{P}<0.001$, by 1-way ANOVA with Tukey's multiple comparisons test. (E and $\mathbf{F}$ ) Cleaved caspase-3 staining in CSC 387 (E) and 3565 (F) after HELLS knockdown. Three independent experiments were performed. Cleaved caspase-3 signals are shown as red and DAPI as blue. Scale bars: $20 \mu \mathrm{m}$. (C) Quantification of cleaved caspase-3+ cells in CSC 387 (top) and 3565 (bottom) after HELLS knockdown. Data are presented as mean \pm SD. ${ }^{* *} P<0.001$, by 1-way ANOVA with Tukey's multiple comparisons test. For GSC 387: shCONT, $n=7$; shHELLS.1744, $n=8$; shHELLS.1308, $n=8$. For GSC 3565: shCONT, $n=7$; shHELLS.1744, $n=7$; shHELLS.1308, $n=7$. (H and I) Immunoblot for cleavage of PARP (H) and caspase-3 (I) in GSC 387, 3565, and 3691 after HELLS knockdown. Three independent experiments were performed. 
A

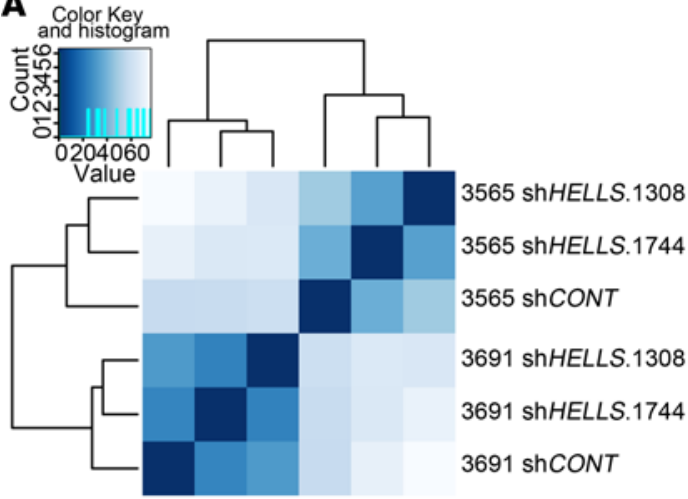

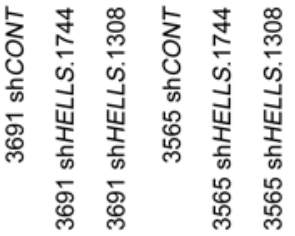

D

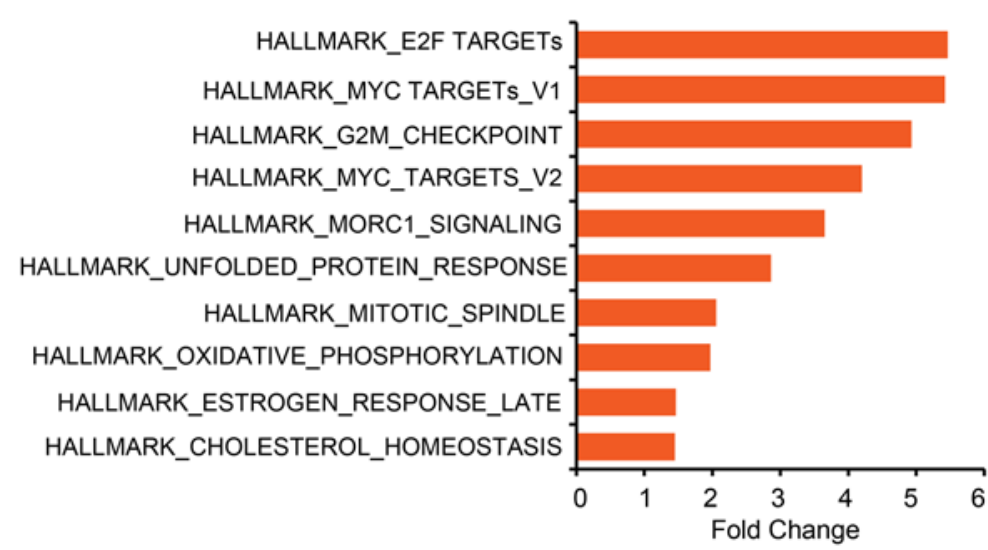

$\mathbf{F}$

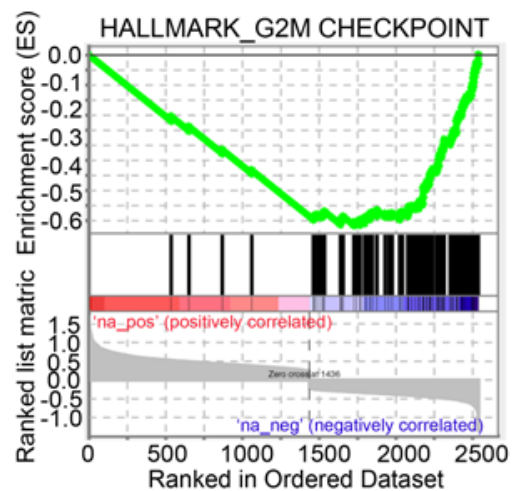

B

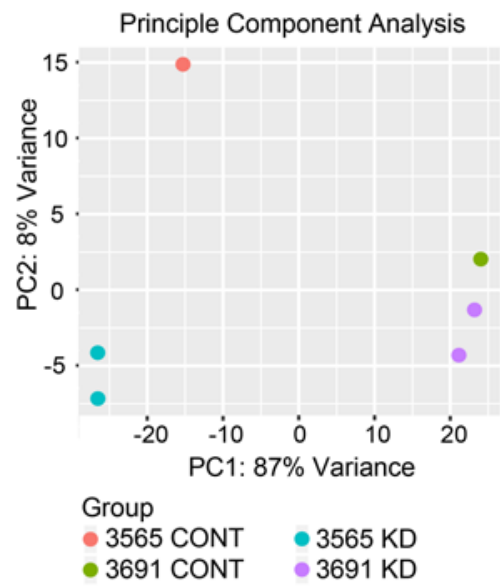

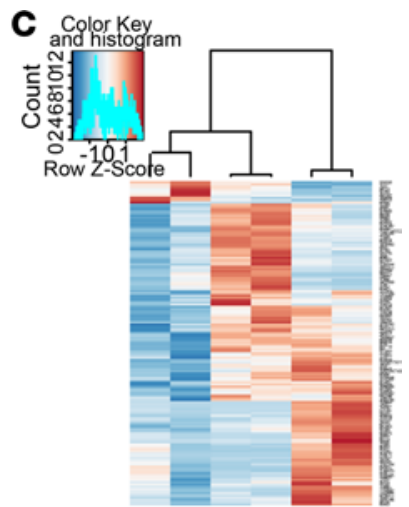

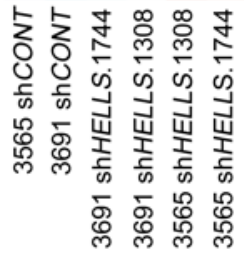

E

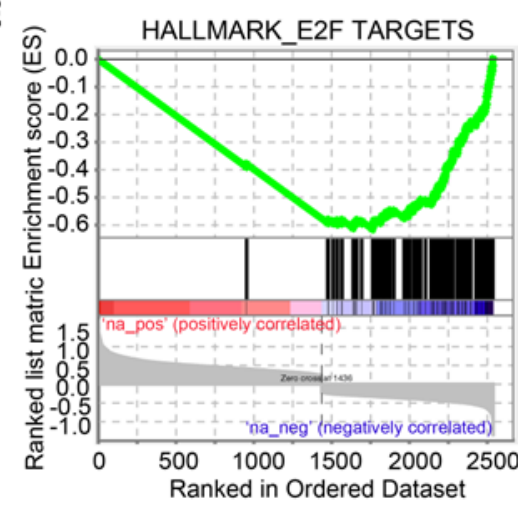

H

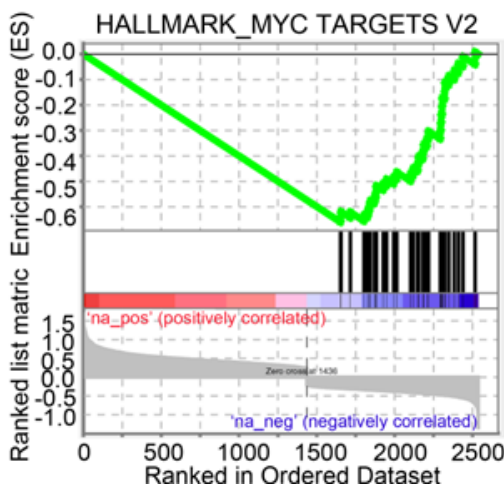

Figure 7. HELLS expression correlates with expression of E2F3 and MYC mRNA and downstream targets. (A) GSC 3565 and 3691 were transduced with either shCONT or 1 of 2 nonoverlapping sHELLS; they then underwent expression analysis by RNA-seq. Unbiased clustering is shown. (B) Principal component analysis of RNA-seq data from A in GSC 3565 and 3691 after HELLS knockdown. (C) Heatmap of the differentially expressed genes in GSC 3565 and 3691 after HELLS knockdown in A. (D) GSEA for the differentially expressed genes obtained from GSC models 3565 and 3691 after HELLS knockdown in A. (E-H) Enrichment plots for E2F targets (E), $\mathrm{G}_{2} / \mathbf{M}$ checkpoint $(\mathbf{F})$, and MYC targets ( $\mathbf{G}$ and $\mathbf{H}$ ) derived from RNA-seq data in $\mathbf{A}$.

formly (Supplemental Figure 9, A-F). Beyond brain cancers, HELLS informed poor prognosis of other cancers, including cancers of the breast, lung, stomach, liver, and renal papillary cells (Supplemental Figure 9, G-L). Collectively, these results support HELLS as a clinically relevant target in glioblastoma and potentially other cancers driven by cancer stem cells. 
A
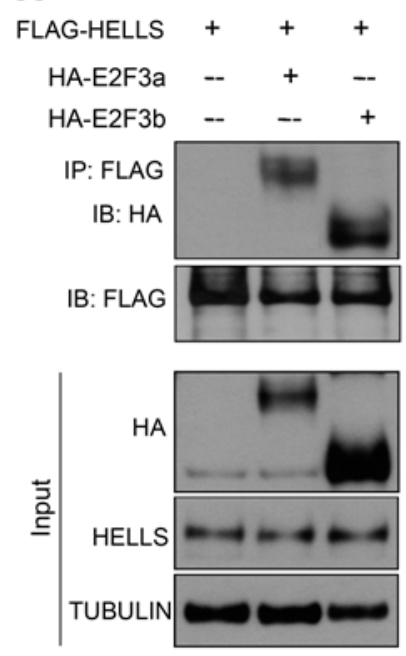

D

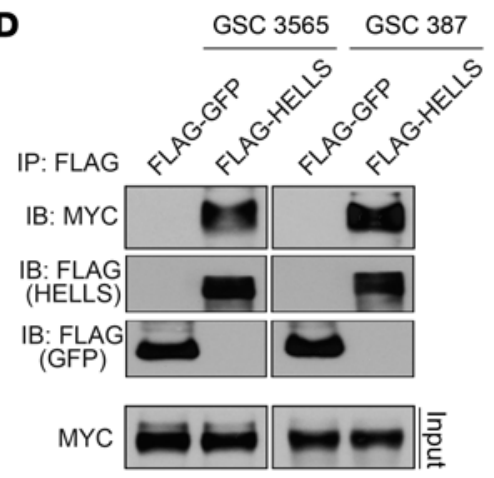

E
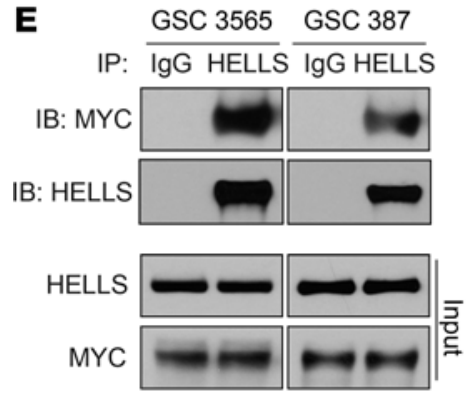

\section{B}

C
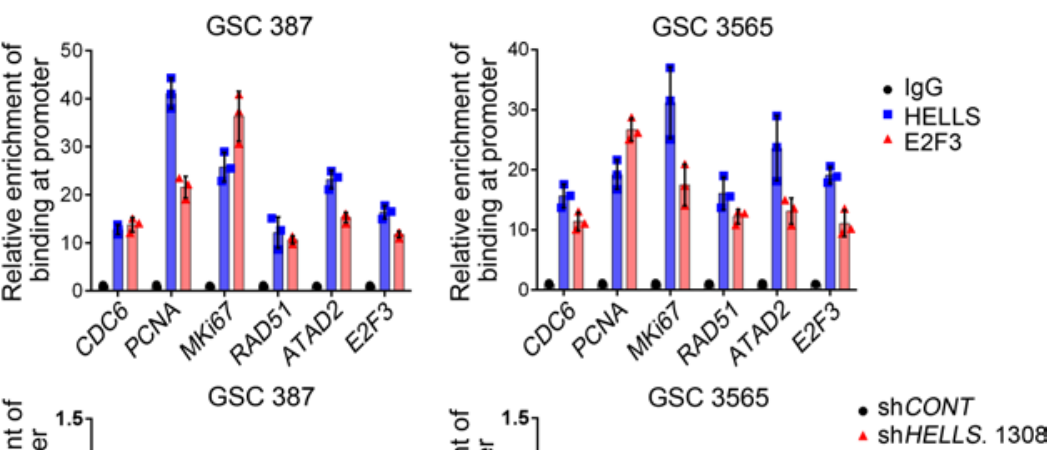

A shHELLS. 1308

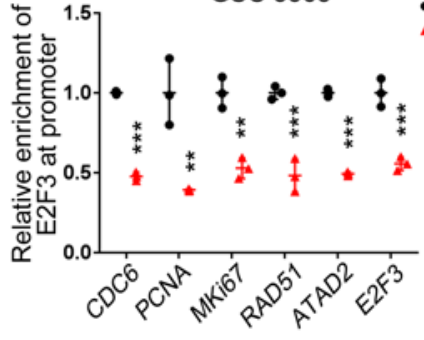

$\mathbf{F}$

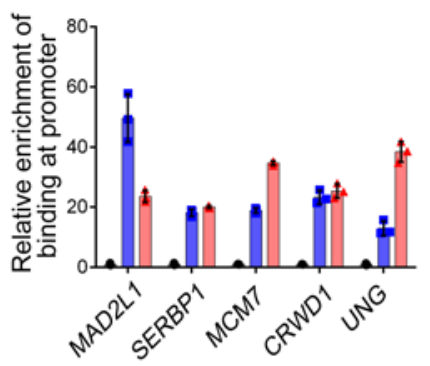

G

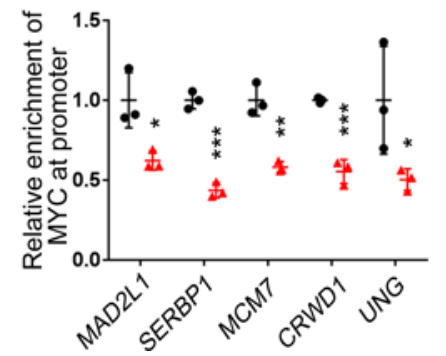

GSC 3565

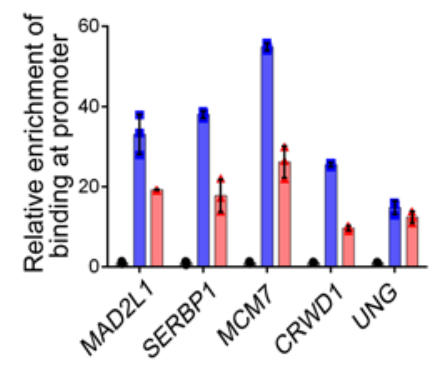

- $\lg G$

- HELLS

$\triangle \mathrm{MYC}$

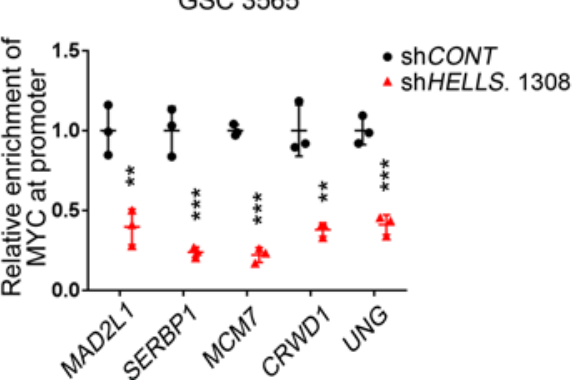

Figure 8. HELLS interacts with E2F3 and MYC to regulate gene expression. (A) GSC 3565 cells were transduced with FLAG-HELLS and either HA-E2F3a or HA-E2F3b and then underwent whole cell lysis. Immunoprecipitation for HELLS was performed on the lysates with an anti-FLAC antibody, which were then resolved by SDS-PAGE, and immunoblotting was performed with an anti-HA antibody. Inputs are indicated. Results are typical of 3 independent experiments. (B) GSC 387 and 3565 underwent ChIP followed by PCR (ChIP-qPCR) using antibodies for IgG control, HELLS, or E2F3. Primers specific for previously described E2F3 targets were used (ref. 32). Data are presented as mean \pm SD. Three independent experiments were performed. (C) ChIP-qPCR analysis for binding of E2F3 to its targets gene in GSC 387 and 3565 after HELLS knockdown. Data are presented as mean \pm SD. ${ }^{* *} P<0.01,{ }^{* * *} P<0.001$, by $t$ test. Three independent experiments were performed. (D) GSC 387 and 3565 were transduced with either FLAG-HELLS or FLAG-GFP and then lysed. An anti-FLAC antibody was used for immunoprecipitation, and immunoblotting was performed with an anti-MYC antibody. Inputs are indicated. Three independent experiments were performed. (E) Endogenous coimmunoprecipitation experiments to consider HELLS and MYC binding were performed in GSC 387 and 3565. Whole cell lysates were collected and subjected to immunoprecipitation with an anti-HELLS or IgG control antibody and then immunoblotting was performed with an anti-MYC antibody. Inputs are indicated. Three independent experiments were performed. (F) ChIP-qPCR analysis for binding of HELLS and MYC in GSC 387 and 3565 . Primers specific for MYC targets were used (ref. 32). IgG was used as control. Data are presented as mean \pm SD. Three independent experiments were performed. (G) ChIP-qPCR analysis for binding of MYC to its targets gene in CSC 387 and 3565 after HELLS knockdown. Data are presented as mean $\pm \mathrm{SD}$. ${ }^{*} P<0.05$, ${ }^{* *} P<0.01,{ }^{* *} P<0.001$, by $t$ test. Three independent experiments were performed. 


\section{Discussion}

Cancer is a disease of uncontrolled cell proliferation (33). Proliferation is a histologic marker of tumor grade, suggesting that identifying drivers of tumor cell proliferation is important to understanding tumor biology. While genetic mutations are generally believed to be the drivers of tumorigenesis, emerging evidence showed that epigenetic regulators are also involved in tumorigenesis and progression $(17,18)$. Normal neural stem cells (NSCs) are often quiescent, leading to a focus on quiescence in GSCs (34-36). However, the stem cell-like compartment in gliomas also harbors the proliferating cellular fraction, suggesting that GSCs are heterogeneous in their regulation of the cell cycle. In tissues outside of the brain, including the gut and skin, stem cells can be dichotomized into at least 2 fractions with subsets that are quiescent and others that are highly proliferative. Thus, regulatory control of proliferation in GSCs may provide novel therapeutic targets not appreciated in the analysis of bulk tumor cell populations.

Leveraging an in silico discovery approach, we mapped core epigenetic regulators against a gene expression signature for cell cycle progression, revealing a restricted set of molecules that were highly correlated with cell cycle regulators (Figure 1, A and B). Prominently, top targets included EZH2 and KDM1A, which are highly expressed in cycling cells and important for GSC maintenance, suggesting utility of the discovery methodology $(21,37,38)$. HELLS ranked as another molecule tightly correlated with cycling gene expression in glioblastoma. As a member of the SWI2/SNF2 superfamily, HELLS is required for de novo DNA methylation of repeat sequences and silencing of $H O X$ gene expression during development by interacting with DNMT3B and PRC1 components $(39,40)$. Notably, both DNMT1 and DNMT3B tightly correlated with cell cycle regulators in glioblastoma in our analysis.

HELLS has been reported to serve important functions in the regulation of normal stem cells. HELLS regulates NSC proliferation and self-renewal by exerting epigenetic regulation at key regulators of NSC fate during development (41). HELLS also regulates expression of genes important for embryonic stem cell maintenance by controlling the DNA methylation patterns at stem cell genes (42). Parallel to these findings, deletion of HELLS resulted in a lethal phenotype and the mice died perinatally, indicating the importance of HELLS for embryonic development (43).

Here, we found that HELLS expression was associated with glioma progression and highly expressed in glioblastoma. Recently, HELLS was linked to glioma biology as a downstream target of LRP6-GSK3 $\beta$ E2F1 signaling (44). This prior report investigated upstream regulation of HELLS in cell lines, whereas we found that HELLS was predominantly expressed in GSCs and investigated downstream signaling, revealing that HELLS interacts with E2F3 and MYC in GSCs to regulate cycling gene expression to maintain GSC proliferation and self-renewal. HELLS has been reported to interact with E2F3 in cell lines from other cancer types (32), but these prior studies did not find the reduction of E2F3 enrichment at its target gene promoters that we found in a cancer stem cell population, suggesting a context-dependent regulation. Similar to previous the study, we also found that E2F3 overexpression could not fully compensate for loss of HELLS in GSCs. The complexity of transcriptional regulation downstream from HELLS likely derives from its multiple interactions, including with MYC to activate gene expression and with DNMT and the PRC1 complex to silence gene expression or heterochromatic regions $(39,40)$. Thus, HELLS is necessary for cell cycle progression, but it is unlikely that any single interactor is sufficient for the full spectrum of biologic effects of HELLS. Future studies will determine the selectivity of HELLS coordination to activate some genes while repressing others. HELLS knockdown decreased the expression of E2F3 targets and MYC targets and induced the replication stress followed by DSBs, which caused cell cycle arrest and apoptosis in GSCs. Supporting our observation about DNA damage after HELLS knockdown, it was shown recently in HEK293 that HELLS knockout caused DSBs at centromeric, pericentromeric, and telomeric repeats, which was speculated to be caused by replication stress; however, there is no evidence supporting this (45). Other chromatin remodelers were reported to play roles in DNA damage repair (27); however, our findings, together with the results of Unoki and coworkers suggest that HELLS may also serve functions in DNA damage responses. We found that the oncogenic roles of HELLS in glioblastoma are likely mediated through interactions with E2F3 and MYC. Given the wide overexpression of E2F3 and MYC in many different cancers, the interaction between HELLS and E2F3 or MYC may help us to better understand the molecular mechanisms that underlie the aberrant cell cycle control in multiple cancer types.

Core drivers of cellular proliferation in oncology, such as MYC and E2F family members, have proven challenging to drug. As a key mediator of proliferation, HELLS may offer a tractable molecular target to disrupt the functional effects of MYC and E2F3. As a helicase, HELLS contains an enzymatic function that 
A

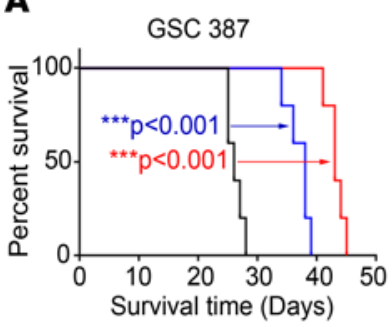

C
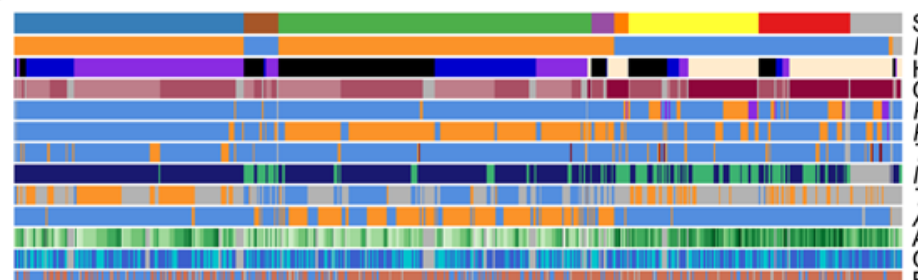

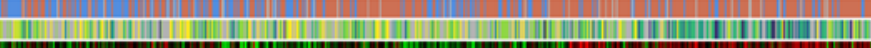
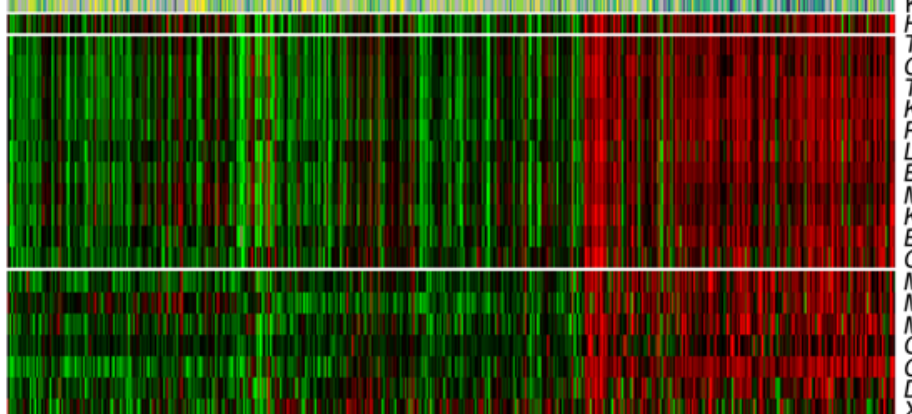

D

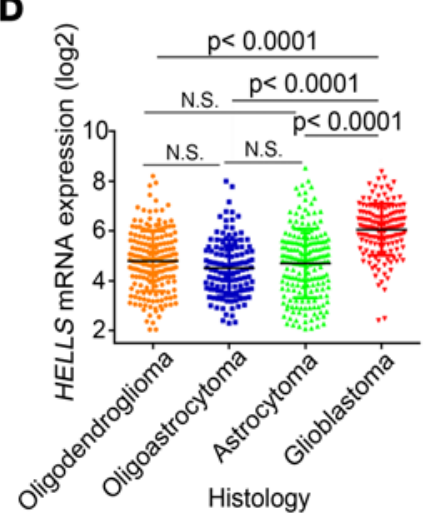

E

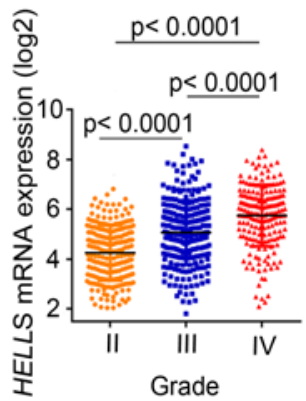

B

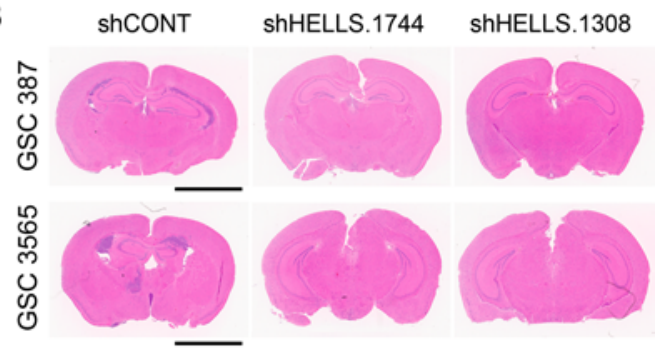

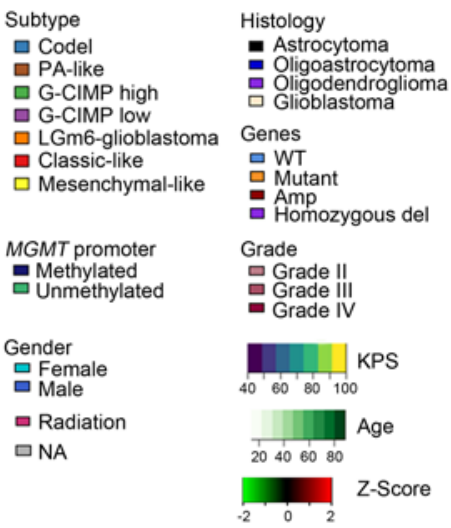

Subtype

$\square$ Codel

$\square \mathrm{G}-\mathrm{CIMP}$

Classic-like

GMT promoter

ERT promoter

Age

Gender

Radiation

RPS

PP2A

CENPF

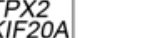

PRC1 E2F3

IUNB1 targets

MKi67

KIF11

EZH2

MCM3

MCM4

MCM5 MYC

CDK4 targets

CDC20

\section{F}

Glioblastoma + Low grade glioma

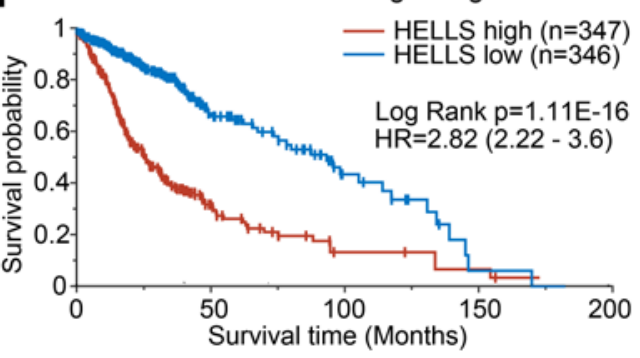

\begin{tabular}{|c|c|c|c|c|c|c|}
\hline \multirow{2}{*}{ High } & $\mathrm{IDH}$ w & 182 & 3 & 1 & 1 & 0 \\
\hline & IDH mut & 165 & 27 & 5 & 4 & 0 \\
\hline \multirow{2}{*}{ Low } & $\mathrm{IDH}$ wt & 72 & 1 & 0 & 0 & 0 \\
\hline & IDH mut & 274 & 55 & 14 & 2 & 0 \\
\hline
\end{tabular}

Figure 9. HELLS is required for in vivo glioblastoma growth and informs prognosis. (A) Kaplan-Meier survival curves of immunocompromised mice bearing intracranial CSC 387 and 3565 transduced with either 1 of 2 shRNAs targeting HELLS or nontargeting control. ${ }^{* * *} P<0.001$, by Mantel-Cox log-rank test. $n=5$. (B) Representative images of H\&E-stained brain sections 20 days after transplanting CSC 387 and 3565 transduced with either 1 of 2 shRNAs targeting HELLS or nontargeting control. Scale bars: $3 \mathrm{~mm}$. (C) RNA-seq, whole exome, and clinical phenotype data were aggregated from TCCA glioblastoma (CBM) and low-grade glioma (LGG) data sets to visualize the expression patterns of HELLS, E2F3 targets, and MYC targets across glioma. "Codel," codeletion of chromosomes 1p and 19q; PA-like, pilocytic astrocytoma-like; CIMP, glioma-CpG island methylator phenotype; LGm6-GBM, a subgroup of glioma enriched for histologic low-grade gliomas that also contains a subset of tumors with GBM-defining histologic criteria; KPS, Karnofsky performance status. (D) HELLS expression in patients with oligodendroglioma, oligoastrocytoma, astrocytoma, and glioblastoma. Data are presented as mean \pm SD. $P$ values were determined by 1-way ANOVA with Tukey's multiple comparisons test. (E) HELLS expression across glioma tumor grades. Data are presented as mean \pm SD. $P$ values were determined by 1-way ANOVA with Tukey's multiple comparisons test. (F) Survival curves of glioma patients with higher and lower HELLS expression. Significance was determined by Mantel-Cox log-rank testing. The distribution of IDH WT and mutant patients in both the HELLS ${ }^{\text {hi }}$ (red) and the HELLS ${ }^{10}$ (blue) group at the indicated time points is listed at bottom.

is amenable to inhibition. Pharmacologic inhibitors have been developed against other helicases, including WRN and DDX3, for indications that include oncology and infectious diseases (46-48). While pharmacologic inhibitors of HELLS have not yet been reported, the development of such agents may have substantial value, not only as monotherapy, but also in combination with conventional therapy and molecularly targeted 
therapy. Other chromatin regulators have been reported as biomarkers of sensitivity to PARP inhibitors in other cancers (49), and targeting the DDX3 helicase induces synthetic lethality with PARP in BRCA1-proficient breast cancer (50). We previously demonstrated that GSCs are highly responsive to PARP inhibitors and that BRCA1 serves an oncogenic function in glioblastoma, suggesting that targeting HELLS in combination with PARP inhibitors could offer a potent therapeutic paradigm against the most resistant tumor cell populations in glioblastoma $(51,52)$. Collectively, our results lay the foundation for the development of a potentially novel molecular targeting approach for the treatment of one of the most lethal human malignancies.

\section{Methods}

Derivation of GSCs, nonmalignant brain cultures, and NSCs. Patient-derived GSCs, GSC 387 and GSC 3691, were generated by our laboratory and transferred via a MTA from Duke University. GSC 3565 was derived by our lab with human specimens from Case Western Reserve University. Nonmalignant brain cultures (NM176, NM177, and NM263) were derived by our lab with surgical tissue from human epilepsy resection specimens. Human NSC line NSC11 derived from human iPS cells was purchased from ALSTEM. ENSA cells (ENStem-A, Millipore) are human embryonic stem-derived neural progenitor cells. HNP1 Human Neural Progenitors (ArunA Biomedical Inc.) are fully differentiated and derived as adherent cells from the hESC WA09 line. All NSCs were cultured in Neurobasal media (Invitrogen) supplemented with B27 (Invitrogen), EGF, and basic FGF (20 ng/ml each; R\&D Systems). To decrease the incidence of cell culture-based artifacts, patient-derived xenografts were produced and propagated as a renewable source of tumor cells for study. All cells were thawed within 1 month of these experimental procedures.

In vivo tumorigenesis. Intracranial xenografts were generated by transplanting $5 \times 10^{4}$ human-derived GSCs into the right cerebral cortex of NSG (NOD.Cg-Prkdcscid I12rgtm1Wj1/SzJ, The Jackson Laboratory) mice at a depth of $3.5 \mathrm{~mm}$. Healthy male and female mice on a NSG background, at 4-6 weeks old, were randomly selected and used in this study. All mice were maintained in a 12-hour light/12-hour dark cycle by animal husbandry staff at University of California, San Diego, with no more than 5 mice per cage. Housing conditions and animal status were supervised by a veterinarian. Animals in experiments were monitored every day until neurological signs were observed, at which point they were sacrificed. Neurological signs or signs of morbidity included hunched posture, gait changes, lethargy, and weight loss. Brains were harvested and fixed in $4 \%$ formaldehyde overnight. H\&E staining was performed on sections for histological analysis. In parallel survival experiments, mice were observed until the development of neurological signs.

Tumor dissociation and GSC culture. Xenografted tumors were dissociated using a Papain Dissociation System (Worthington) according to the manufacturer's instructions. Cells were then cultured in Neurobasal medium supplemented with 2\% B27, 1\% L-glutamine, 1\% sodium pyruvate, 1\% penicillin/ streptomycin, $10 \mathrm{ng} / \mathrm{ml}$ basic FGF, and $10 \mathrm{ng} / \mathrm{ml} \mathrm{EGF}$ for at least 6 hours to recover expression of surface antigens. As cell surface markers are not uniformly informative for enriching for GSCs, we utilized a combination of functional criteria to validate GSCs. Both GSCs and DGCs were derived immediately following dissociation or following transient xenograft passage in NSG mice using prospective sorting followed by assays to confirm stem cell marker expression, sphere formation, and secondary tumor initiation. Cells were labeled with the CD133/2(293C)-Allophycocyanin (APC) antibody kit (Miltenyi Biotec, 130098826), and the CD133+ cells were sorted and analyzed by flow cytometry. CD $133^{+}$cells were cultured in NBM-B27 medium containing $20 \mathrm{ng} / \mathrm{ml}$ of both EGF (R\&D Systems, 236-EG-01M) and recombinant human basic FGF (R\&D Systems, 4114-TC-01M) before treatment and analysis. GSC phenotypes were validated by expression of stem cell markers (SOX2 and OLIG2) and functional assays of self-renewal. Corresponding differentiated glioma cells were obtained by culturing the GSCs in DMEM medium supplemented with 10\% FBS for at least 2 weeks.

Proliferation and neurosphere formation assay. Cell proliferation experiments were carried out by plating cells of interest at a density of $10^{3}$ cells per well in a 96-well plate with at least 3 replicates. Relative cell numbers were measured by CellTiter-Glo (Promega) assay in a time-course manner. All data were normalized to day 0 and presented as mean \pm SD to show the relative proliferation rates of cells after different treatment. Neurosphere formation was conducted by in vitro limiting dilution assay, as previously reported (53). Briefly, decreasing numbers of cells per well (200, 100, 50, 25, and 10 cells) were plated into 96-well plates. The presence and number of neurospheres in each well were recorded 7 days after plating. Extreme limiting dilution analysis was performed using available software (http://bioinf.wehi. edu.au/software/elda), as previously described (54). 
Immunofluorescent staining. Cells were plated on ground coverslips (Bellco Glass Inc.) and then were fixed with $4 \%$ paraformaldehyde for 10 minutes at room temperature, followed by blocking with $5 \%$ normal goat serum in PBST for 20 minutes at room temperature. After that, cells were incubated with anti-HELLS (Millipore, catalog ABD41, 1:500), anti-HELLS (Santa Cruz, catalog sc-46665, 1:200), anti-Ki67 (DAKO, catalog M7240, 1:100), anti-cleaved caspase-3 (CST, catalog 9664, 1;500), anti- $\gamma \mathrm{H} 2 \mathrm{AX}$ (Abcam, catalog ab11174, 1:1000), and anti-pRPA (Abcam, catalog ab61065, 1:500) antibodies overnight at $4^{\circ} \mathrm{C}$. After washing 3 times with PBS, cells were incubated with anti-rabbit or anti-mouse IgGs conjugated with Alexa Fluor 488 or Alexa Fluor 568 (Invitrogen, 1:500) and costained with DAPI for 2 hours at room temperature. Slides were then mounted with Fluoromount-G (SouthernBiotech Inc.) and processed to imaging with microscope or confocal.

ChIP analysis. GSCs in culture medium were fixed with $1 \%$ paraformaldehyde at room temperature for 10 minutes, followed by $0.125 \mathrm{mM}$ glycine treatment for 5 minutes at room temperature. After washing with ice-cold PBS, collected cells were processed to release nuclei by suspending in Cell Lysis buffer (10 $\mathrm{mM}$ Tris- $\mathrm{HCl}, 10 \mathrm{mM} \mathrm{NaCl}, 1.5 \mathrm{mM} \mathrm{MgCl}_{2}, \mathrm{pH}$ 8.1) containing protease inhibitors and kept on ice for 15 minutes. Then, nuclei were collected by spinning down at $1000 \mathrm{~g}$ for 5 minutes at $4^{\circ} \mathrm{C}$ and suspended by Nuclear Lysis buffer (1\% SDS, 10 mM EDTA, 50 mM Tris-HCl, pH 8.1) containing protease inhibitors. Genomic DNA was then sheared by sonication to a length between $200 \mathrm{bp}$ and $1000 \mathrm{bp}$. After centrifuging at $15,294 \mathrm{~g}$ for 10 minutes at $4^{\circ} \mathrm{C}, 100 \mu \mathrm{l}$ of the supernatant was taken and mixed with $900 \mu \mathrm{ChIP}$ dilution buffer (0.01\% SDS, 1.1\% Triton X-100, 1.2 mM EDTA, $16.7 \mathrm{mM}$ Tris-HCl, pH 8.1, $167 \mathrm{mM}$ $\mathrm{NaCl}$ ), $5 \mu$ Protein A magnetic beads, and $5 \mu 1$ Protein $\mathrm{G}$ magnetic beads and $2 \mu \mathrm{g}$ anti-HELLS (Millipore), anti-FLAG (MilliporeSigma), or anti-E2F3 (Thermo Fisher Scientific) antibodies. Normal rabbit or mouse IgGs (Santa Cruz) were used as controls. After rotating at $4^{\circ} \mathrm{C}$ overnight, the magnetic beads were washed with Low Salt Wash buffer, High Salt Wash buffer, LiCl Wash buffer, and TE Wash buffer (all from EZ-Magna ChIP A/G kit, Millipore) 5 minutes for each. The beads were then suspended in ChIP Elution buffer supplemented with Proteinase $\mathrm{K}$ and incubated at $62^{\circ} \mathrm{C}$ for 2 hours with shaking, followed by incubation at $95^{\circ} \mathrm{C}$ for 10 minutes. Then, the supernatant was processed for DNA purification with the PCR purification kit (Qiagen), and the resulting DNA was used for qPCR analysis or sequencing.

Coimmunoprecipitation analysis. GSCs expressing FLAG-HELLS alone, FLAG-HELLS and HA-E2F3a, or FLAG-HELLS and HA-E2F3b were collected and washed twice with ice-cold PBS. Cell pellets were suspended with NP40 lysis buffer (Research Products International) supplemented with protease inhibitors and kept on ice for 30 minutes. Cell lysates were centrifuged at $15,294 \mathrm{~g}$ for 10 minutes at $4^{\circ} \mathrm{C}$, and 50 $\mu 1$ supernatant was taken as input while the rest was mixed with anti-FLAG M2 magnetic beads (MilliporeSigma) and rotated at $4^{\circ} \mathrm{C}$ for overnight. Then, the beads were washed twice with NP40 lysis buffer and twice with PBS, followed by boiling in $40 \mu \mathrm{SDS}$ Laemmli loading buffer at $95^{\circ} \mathrm{C}$ for 5 minutes. The resulted supernatant was used for Western blot analysis.

Flow cytometry-based EdU and annexin V staining. EdU staining was done according to the protocol provided with the Click-iT Plus EdU Flow Cytometry Assay Kit (Invitrogen). Briefly, cells were cultured in $10 \mu \mathrm{M}$ EdU for 2 hours, and then collected cells were washed with 1\% BSA in PBS followed by fixation at room temperature for 15 minutes. The fixed cells were washed with $1 \%$ BSA in PBS again and suspended in permeabilization buffer for 15 minutes by avoiding light. The cell suspension was mixed with Click-iT plus reaction cocktail (Invitrogen) and was incubated at room temperature for 30 minutes by avoiding light. The stained cells were washed and then suspended with saponin-based permeabilization and wash reagent. After adding DAPI to the cell suspension, the stained cells were processed to flow cytometry analysis.

Annexin V staining was conducted based on the protocol provided with the Alexa Fluor 488 Annexin V/Dead Cell Apoptosis Kit (Invitrogen). Briefly, harvested cells were washed with ice-cold PBS. After washing, appropriate numbers of cells were suspended in $100 \mu 1$ annexin-binding buffer supplemented with Alexa Fluor 488 annexin V and propidium iodide followed by 15-minute incubation at room temperature. After incubation, $400 \mu 1$ annexin-binding buffer was added and mixed gently. The stained samples were kept on ice and processed for flow analysis as soon as possible.

Western blotting. Cells were collected and lysed in RIPA buffer containing protease inhibitors and incubated on ice for 30 minutes. Supernatant was then collected by centrifuging the lysates at 15,294 $g$ for 10 minutes at $4^{\circ} \mathrm{C}$. The Bradford assay was used to measure the protein concentration in the supernatant. The protein samples were split into small amounts and then stored at $-80^{\circ} \mathrm{C}$ or processed for immediate use. Immediately afterward, the protein samples were mixed first with SDS Laemmli loading buffer and then boiled for 5 minutes, and equal amounts of protein were used for electrophoresis with PAGE gels. After transferring onto PVDF membranes, 
TBST supplemented with 3.5\% BSA was used for blocking at room temperature for 30 minutes followed by incubating with primary antibodies overnight at $4^{\circ} \mathrm{C}$. The membranes were developed with the SuperSignal West Pico PLUS Chemiluminescent Substrate (Thermo Fisher Scientific) and Autoradiography Film (Denville Scientific Inc.). The developed films were scanned with Epson Perfection V600 Photo (Epson).

$R N A$ and quantitative real-time PCR. Total cellular RNA was isolated by use of Trizol reagent (Invitrogen) and dissolved in RNase-free water. After measuring RNA concentration with Nanodrop 1000 (Thermo Fisher Scientific), cDNA was synthesized with the High-Capacity cDNA Reverse Transcription kit (Life Technology) from $1 \mu$ g total RNA. Quantitative real-time PCR was then performed using the Applied Biosystems 7900HT cycler or Bio-Rad CFX 9600 with SYBR-Green PCR Master Mix (Thermo Fisher Scientific) or the Green Hi-ROX qPCR Kit (Radiant).

Plasmids and lentiviral transduction. Lentiviral clones expressing 2 nonoverlapping shRNAs directed against human HELLS (TRCN0000000307 and TRCN0000000306), human E2F3 (TRCN0000013804 and TRCN0000013807), or a nontargeting control shRNA were obtained from MilliporeSigma. 293FT cells were used to generate lentiviral particles through cotransfection of the packaging vectors pCMV-dR8.2 dvpr and pCI-VSVG using a standard calcium phosphate transfection method in DMEM plus 10\% FBS. Twelve hours after transfection, medium was changed to neurobasal complete medium. Neurobasal complete medium containing lentiviral particles was collected by filtering with a $0.45-\mu \mathrm{M}$ filter (from VWR) for immediate use or stock at $-80^{\circ} \mathrm{C}$.

Patient database bioinformatics. To determine the clinical relevance of patients with glioblastoma, we interrogated TCGA glioblastoma data set (55). Expression levels were categorized into 2 groups with the median value as a cutoff. The survival times and status for patients in both groups were analyzed using the Kaplan-Meier statistical test via log-rank test.

Statistics. Kaplan-Meier survival curves were generated using Prism software (GraphPad), and a logrank test was performed to assess statistical significance between groups. For quantification with more than 2 groups, 1-way ANOVA was used to assess statistical significance with Prism software. For quantification with more than 2 groups and groups that have subgroups, 2-way ANOVA was use to assess statistical significance with Prism software. Student's 2-tailed $t$ test was used to assess statistical significance of studies with only 2 groups. For the limited dilution assay, $\chi^{2}$ test was used for pair-wise differences in stem population frequency. All data are presented as mean $\pm \mathrm{SD}$, and $P<0.05$ was considered to be significant.

Study approval. All mouse experiments were performed under an animal protocol approved by the University of California, San Diego, Institutional Animal Care and Use Committee.

\section{Author contributions}

GZ and ZD initiated and designed the study, acquired and analyzed the data, and wrote/revised the manuscript. RCG analyzed the ChIP-seq data and revised the manuscript. QW provided experimental assistance for intracranial injection. BCP and LJKK analyzed the data and revised the manuscript. XW performed experimental assistance. $\mathrm{ZD}, \mathrm{GZ}, \mathrm{SB}, \mathrm{PH}$, and JNR conceptualized the study and wrote and revised the manuscript, with contributions from all the authors. JNR and PH conceived and designed the study, developed the methodology, analyzed and interpreted the data, wrote and/or revised the manuscript, and provided study supervision and material support.

\section{Acknowledgments}

We appreciate the Histology Core at UCSD for their work on histologic experiments and analysis. We thank Kathrin Muegge and Irina Stancheva for suggestions on the choice of HELLS antibodies. The work was supported by NIH grants (CA217066 to BCP; RCG: CA217065 to RCG; CA203101 to LJKK; NS091080, NS099175 to SB; and CA197718, CA154130, CA169117, CA171652, NS087913, and NS089272 to JNR). $\mathrm{PH}$ is supported by the Danish Cancer Society/Kraeftens Bekaempelses Videnskabelige Udvalg Foundation, Knæk Cancer, and the Novo Nordisk Foundation.

Address correspondence to: Jeremy N. Rich, 2880 Torrey Pines Scenic Drive, La Jolla, California 92037, USA. Phone: 858.822.2703; Email: drjeremyrich@gmail.com. Or to: Petra Hamerlik, Brain Tumor Biology, Danish Cancer Society Research Center, Strandboulevarden 49, DK-2100, Copenhagen, Denmark. Phone: 45.35.35.74.05; Email: pkn@cancer.dk. 
1. Weller M, et al. Glioma. Nat Rev Dis Primers. 2015;1:15017.

2. Ostrom QT, et al. CBTRUS Statistical Report: Primary brain and other central nervous system tumors diagnosed in the United States in 2010-2014. Neuro-oncology. 2017;19(suppl_5):v1-v88.

3. Stupp R, et al. Effects of radiotherapy with concomitant and adjuvant temozolomide versus radiotherapy alone on survival in glioblastoma in a randomised phase III study: 5-year analysis of the EORTC-NCIC trial. Lancet Oncol. 2009;10(5):459-466.

4. Stupp R, et al. Radiotherapy plus concomitant and adjuvant temozolomide for glioblastoma. N Engl J Med. 2005;352(10):987-996.

5. Darmanis S, et al. Single-cell RNA-seq analysis of infiltrating neoplastic cells at the migrating front of human glioblastoma. Cell Rep. 2017;21(5):1399-1410.

6. Meyer M, et al. Single cell-derived clonal analysis of human glioblastoma links functional and genomic heterogeneity. Proc Natl Acad Sci USA. 2015;112(3):851-856.

7. Patel AP, et al. Single-cell RNA-seq highlights intratumoral heterogeneity in primary glioblastoma. Science. 2014;344(6190):1396-1401.

8. Lathia JD, Mack SC, Mulkearns-Hubert EE, Valentim CL, Rich JN. Cancer stem cells in glioblastoma. Genes Dev. 2015;29(12):1203-1217.

9. Galli R, et al. Isolation and characterization of tumorigenic, stem-like neural precursors from human glioblastoma. Cancer Res. 2004;64(19):7011-7021.

10. Singh SK, et al. Identification of human brain tumour initiating cells. Nature. 2004;432(7015):396-401.

11. Bao S, et al. Stem cell-like glioma cells promote tumor angiogenesis through vascular endothelial growth factor. Cancer Res. 2006;66(16):7843-7848.

12. Calabrese C, et al. A perivascular niche for brain tumor stem cells. Cancer Cell. 2007;11(1):69-82.

13. Jin X, et al. Targeting glioma stem cells through combined BMI1 and EZH2 inhibition. Nat Med. 2017;23(11):1352-1361.

14. Bao S, et al. Glioma stem cells promote radioresistance by preferential activation of the DNA damage response. Nature. 2006;444(7120):756-760.

15. Pallini R, et al. Cancer stem cell analysis and clinical outcome in patients with glioblastoma multiforme. Clin Cancer Res. 2008;14(24):8205-8212.

16. Tirosh I, et al. Single-cell RNA-seq supports a developmental hierarchy in human oligodendroglioma. Nature. 2016;539(7628):309-313.

17. Sharma S, Kelly TK, Jones PA. Epigenetics in cancer. Carcinogenesis. 2010;31(1):27-36.

18. Biswas S, Rao CM. Epigenetics in cancer: Fundamentals and Beyond. Pharmacol Ther. 2017;173:118-134.

19. Yang Z, Jones A, Widschwendter M, Teschendorff AE. An integrative pan-cancer-wide analysis of epigenetic enzymes reveals universal patterns of epigenomic deregulation in cancer. Genome Biol. 2015;16:140.

20. Kayaselçuk F, Zorludemir S, Gümürdühü D, Zeren H, Erman T. PCNA and Ki-67 in central nervous system tumors: correlation with the histological type and grade. J Neurooncol. 2002;57(2):115-121.

21. Suvà ML, et al. EZH2 is essential for glioblastoma cancer stem cell maintenance. Cancer Res. 2009;69(24):9211-9218.

22. Lopez-Bertoni H, et al. DNMT-dependent suppression of microRNA regulates the induction of GBM tumor-propagating phenotype by Oct4 and Sox2. Oncogene. 2015;34(30):3994-4004.

23. Umate P, Tuteja N, Tuteja R. Genome-wide comprehensive analysis of human helicases. Commun Integr Biol. 2011;4(1):118-137.

24. Bannister AJ, Kouzarides T. Regulation of chromatin by histone modifications. Cell Res. 2011;21(3):381-395.

25. Creyghton MP, et al. Histone H3K27ac separates active from poised enhancers and predicts developmental state. Proc Natl Acad Sci USA. 2010;107(50):21931-21936.

26. Jenness C, Giunta S, Müller MM, Kimura H, Muir TW, Funabiki H. HELLS and CDCA7 comprise a bipartite nucleosome remodeling complex defective in ICF syndrome. Proc Natl Acad Sci USA. 2018;115(5):E876-E885.

27. Rother MB, van Attikum H. DNA repair goes hip-hop: SMARCA and CHD chromatin remodellers join the break dance. Philos Trans R Soc Lond, B, Biol Sci. 2017;372(1731):20160285.

28. Zou Y, Liu Y, Wu X, Shell SM. Functions of human replication protein A (RPA): from DNA replication to DNA damage and stress responses. J Cell Physiol. 2006;208(2):267-273.

29. Zeman MK, Cimprich KA. Causes and consequences of replication stress. Nat Cell Biol. 2014;16(1):2-9.

30. Humbert PO, Verona R, Trimarchi JM, Rogers C, Dandapani S, Lees JA. E2f3 is critical for normal cellular proliferation. Genes Dev. 2000;14(6):690-703.

31. Tsai SY, et al. Mouse development with a single E2F activator. Nature. 2008;454(7208):1137-1141.

32. von Eyss B, et al. The SNF2-like helicase HELLS mediates E2F3-dependent transcription and cellular transformation. EMBO J. 2012;31(4):972-985

33. Evan GI, Vousden KH. Proliferation, cell cycle and apoptosis in cancer. Nature. 2001;411(6835):342-348.

34. Campos B, et al. Aberrant self-renewal and quiescence contribute to the aggressiveness of glioblastoma. $J$ Pathol. 2014;234(1):23-33.

35. Hoang-Minh LB, et al. Infiltrative and drug-resistant slow-cycling cells support metabolic heterogeneity in glioblastoma. $E M B O$ J. 2018;37(23): e98772.

36. Deleyrolle LP, et al. Evidence for label-retaining tumour-initiating cells in human glioblastoma. Brain. 2011;134(Pt 5):1331-1343

37. Margueron R, et al. Ezh1 and Ezh2 maintain repressive chromatin through different mechanisms. Mol Cell. 2008;32(4):503-518.

38. Sareddy GR, Viswanadhapalli S, Surapaneni P, Suzuki T, Brenner A, Vadlamudi RK. Novel KDM1A inhibitors induce differentiation and apoptosis of glioma stem cells via unfolded protein response pathway. Oncogene. 2017;36(17):2423-2434.

39. Xi S, Zhu H, Xu H, Schmidtmann A, Geiman TM, Muegge K. Lsh controls Hox gene silencing during development. Proc Natl Acad Sci USA. 2007;104(36):14366-14371.

40. Yu W, et al. Genome-wide DNA methylation patterns in LSH mutant reveals de-repression of repeat elements and redundant epigenetic silencing pathways. Genome Res. 2014;24(10):1613-1623.

41. Han Y, Ren J, Lee E, Xu X, Yu W, Muegge K. Lsh/HELLS regulates self-renewal/proliferation of neural stem/progenitor cells Sci Rep. 2017;7(1):1136.

42. Xi S, Geiman TM, Briones V, Guang Tao Y, Xu H, Muegge K. Lsh participates in DNA methylation and silencing of stem cell 
genes. Stem Cells. 2009;27(11):2691-2702.

43. Geiman TM, Muegge K. Lsh, an SNF2/helicase family member, is required for proliferation of mature T lymphocytes. Proc Natl Acad Sci USA. 2000;97(9):4772-4777.

44. Xiao D, et al. Chromatin remodeling factor LSH is upregulated by the LRP6-GSK3 $\beta$-E2F1 axis linking reversely with surviva in gliomas. Theranostics. 2017;7(1):132-143.

45. Unoki M, Funabiki H, Velasco G, Francastel C, Sasaki H. CDCA7 and HELLS mutations undermine nonhomologous end joining in centromeric instability syndrome. J Clin Invest. 2019;129(1):78-92.

46. Tantravedi S, et al. Targeting DDX3 in medulloblastoma using the small molecule inhibitor RK-33. Transl Oncol. 2019;12(1):96-105

47. Datta A, Brosh RM. New insights into DNA helicases as druggable targets for cancer therapy. Front Mol Biosci. $2018 ; 5: 59$.

48. Aggarwal M, Sommers JA, Shoemaker RH, Brosh RM. Inhibition of helicase activity by a small molecule impairs Werner syndrome helicase (WRN) function in the cellular response to DNA damage or replication stress. Proc Natl Acad Sci USA. 2011;108(4):1525-1530.

49. Stoepker C, et al. DNA helicases FANCM and DDX11 are determinants of PARP inhibitor sensitivity. DNA Repair (Amst). 2015;26:54-64.

50. Heerma van Voss MR, et al. Combination treatment using DDX3 and PARP inhibitors induces synthetic lethality in BRCA1-proficient breast cancer. Med Oncol. 2017;34(3):33.

51. Venere M, et al. Therapeutic targeting of constitutive PARP activation compromises stem cell phenotype and survival of glioblastoma-initiating cells. Cell Death Differ. 2014;21(2):258-269.

52. Rasmussen RD, et al. BRCA1-regulated RRM2 expression protects glioblastoma cells from endogenous replication stress and promotes tumorigenicity. Nat Commun. 2016;7:13398.

53. Flavahan WA, et al. Brain tumor initiating cells adapt to restricted nutrition through preferential glucose uptake. Nat Neurosci. 2013;16(10):1373-1382.

54. Hu Y, Smyth GK. ELDA: extreme limiting dilution analysis for comparing depleted and enriched populations in stem cell and other assays. J Immunol Methods. 2009;347(1-2):70-78.

55. Bowman RL, Wang Q, Carro A, Verhaak RG, Squatrito M. GlioVis data portal for visualization and analysis of brain tumor expression datasets. Neuro-oncology. 2017;19(1):139-141. 